\title{
Combined QCD and electroweak analysis of HERA data
}

H. Abramowicz, ${ }^{25, \mathrm{~s}}$ I. Abt,${ }^{20}$ L. Adamczyk, ${ }^{8}$ M. Adamus, ${ }^{31}$ S. Antonelli, ${ }^{2}$ V. Aushev,${ }^{17}$ O. Behnke, ${ }^{10}$ U. Behrens, ${ }^{10}$ A. Bertolin, ${ }^{22}$ S. Bhadra, ${ }^{33}$ I. Bloch, ${ }^{11}$ E. G. Boos, ${ }^{15}$ I. Brock,${ }^{3}$ N. H. Brook,${ }^{29}$ R. Brugnera, ${ }^{23}$ A. Bruni, ${ }^{1}$ P. J. Bussey,${ }^{12}$ A. Caldwell, ${ }^{20}$ M. Capua, ${ }^{5}$ C. D. Catterall, ${ }^{33}$ J. Chwastowski, ${ }^{7}$ J. Ciborowski,${ }^{30, t}$ R. Ciesielski, ${ }^{10, e}$ A. M. Cooper-Sarkar, ${ }^{21}$ M. Corradi, ${ }^{1, a}$ R. K. Dementiev, ${ }^{19}$ R. C. E. Devenish, ${ }^{21}$ S. Dusini, ${ }^{22}$ B. Foster, ${ }^{13, k}$ G. Gach, ${ }^{8}$ E. Gallo, ${ }^{13,1}$ A. Garfagnini, ${ }^{23}$ A. Geiser, ${ }^{10}$ A. Gizhko, ${ }^{10}$ L. K. Gladilin, ${ }^{19}$ Yu. A. Golubkov, ${ }^{19}$ G. Grzelak, ${ }^{30}$ M. Guzik, ${ }^{8}$ C. Gwenlan, ${ }^{21}$ W. Hain, ${ }^{10}$ O. Hlushchenko, ${ }^{17}$ D. Hochman, ${ }^{32}$ R. Hori, ${ }^{14}$ Z. A. Ibrahim, ${ }^{6}$ Y. Iga, ${ }^{24}$ M. Ishitsuka, ${ }^{26}$ F. Januschek, ${ }^{10, \mathrm{f}}$ N. Z. Jomhari, ${ }^{6}$ I. Kadenko, ${ }^{17}$ S. Kananov, ${ }^{25}$ U. Karshon, ${ }^{32}$ P. Kaur, ${ }^{4, b}$ D. Kisielewska, ${ }^{8}$ R. Klanner, ${ }^{13}$ U. Klein, ${ }^{10, g}$ I. A. Korzhavina, ${ }^{19}$ A. Kotański, ${ }^{9}$ U. Kötz, ${ }^{10}$ N. Kovalchuk, ${ }^{13}$ H. Kowalski, ${ }^{10}$ B. Krupa ${ }^{7}$ O. Kuprash, ${ }^{10, h}$ M. Kuze, ${ }^{26}$ B. B. Levchenko, ${ }^{19}$ A. Levy, ${ }^{25}$ S. Limentani, ${ }^{23}$ M. Lisovyi, ${ }^{10,}$ E. Lobodzinska, ${ }^{10}$ B. Löhr, ${ }^{10}$ E. Lohrmann, ${ }^{13}$ A. Longhin, ${ }^{22, r}$ D. Lontkovskyi, ${ }^{10}$

O. Yu. Lukina, ${ }^{19}$ I. Makarenko, ${ }^{10}$ J. Malka, ${ }_{10}$ A. Mastroberardino, ${ }^{5}$ F. Mohamad Idris, ${ }^{6, d}$ N. Mohammad Nasir, ${ }^{6}$ V. Myronenko, ${ }^{10}$ K. Nagano, ${ }^{14}$ T. Nobe, ${ }^{26}$ R. J. Nowak, ${ }^{30}$ Yu. Onishchuk, ${ }^{17}$ E. Paul, ${ }^{3}$ W. Perlański, ${ }^{30, u}$ N. S. Pokrovskiy, ${ }^{15}$ A. Polini, ${ }^{1}$ M. Przybycień, ${ }^{8}$ P. Roloff, ${ }^{10, j}$ M. Ruspa,${ }^{28}$ D. H. Saxon ${ }^{12}$ M. Schioppa ${ }^{5}$ U. Schneekloth,${ }^{10}$

T. Schörner-Sadenius, ${ }^{10}$ L. M. Shcheglova, ${ }^{19}$ R. Shevchenko, ${ }_{10,0, p}^{17}$ O. Shkola ${ }^{17}$ Yu. Shyrma ${ }^{16}$ I. Singh,${ }^{4, c}$ I. O. Skillicorn, ${ }^{12}$ W. Słomiński, ${ }^{9}$ A. Solano, ${ }^{27}$ L. Stanco, ${ }^{22}$ N. Stefaniuk, ${ }^{10}$ A. Stern, ${ }^{25}$ P. Stopa, ${ }^{7}$ J. Sztuk-Dambietz, ${ }^{13, \mathrm{f}}$ E. Tassi, ${ }^{5}$ K. Tokushuku, ${ }^{14, \mathrm{~m}} \mathrm{~J}$. Tomaszewska, ${ }^{30, \mathrm{v}} \mathrm{T}$. Tsurugai ${ }^{18} \mathrm{M}$. Turcato ${ }^{13, \mathrm{f}}$ O. Turkot ${ }^{10}{ }^{10}$ T. Tymieniecka ${ }^{31}$ A. Verbytskyi, ${ }^{20}$ W. A. T. Wan Abdullah, ${ }^{6}$ K. Wichmann, ${ }^{10}$ M. Wing, ${ }^{29}$ S. Yamada, ${ }^{14}$ Y. Yamazaki, ${ }^{14, n}$ N. Zakharchuk, ${ }^{17, q}$ A. F. Żarnecki, ${ }^{30}$ L. Zawiejski, ${ }^{7}$ O. Zenaiev, ${ }^{10}$ B. O. Zhautykov, ${ }^{15}$ and D. S. Zotkin ${ }^{19}$

(ZEUS Collaboration)

\author{
${ }^{1}$ INFN Bologna, Bologna, Italy \\ ${ }^{2}$ University and INFN Bologna, Bologna, Italy \\ ${ }^{3}$ Physikalisches Institut der Universität Bonn, Bonn, Germany \\ ${ }^{4}$ Panjab University, Department of Physics, Chandigarh, India \\ ${ }^{5}$ Calabria University, Physics Department and INFN, Cosenza, Italy \\ ${ }^{6}$ National Centre for Particle Physics, Universiti Malaya, 50603 Kuala Lumpur, Malaysia \\ ${ }^{7}$ The Henryk Niewodniczanski Institute of Nuclear Physics, Polish Academy of Sciences, \\ Krakow, Poland \\ ${ }^{8}$ AGH-University of Science and Technology, Faculty of Physics and Applied Computer Science, \\ Krakow, Poland \\ ${ }^{9}$ Department of Physics, Jagellonian University, Krakow, Poland \\ ${ }^{10}$ Deutsches Elektronen-Synchrotron DESY, Hamburg, Germany \\ ${ }^{11}$ Deutsches Elektronen-Synchrotron DESY, Zeuthen, Germany \\ ${ }^{12}$ School of Physics and Astronomy, University of Glasgow, Glasgow, United Kingdom \\ ${ }^{13}$ Hamburg University, Institute of Experimental Physics, Hamburg, Germany \\ ${ }^{14}$ Institute of Particle and Nuclear Studies, KEK, Tsukuba, Japan \\ ${ }^{15}$ Institute of Physics and Technology of Ministry of Education and Science of Kazakhstan, \\ Almaty, Kazakhstan \\ ${ }^{16}$ Institute for Nuclear Research, National Academy of Sciences, Kyiv, Ukraine \\ ${ }^{17}$ Department of Nuclear Physics, National Taras Shevchenko University of Kyiv, \\ Kyiv, Ukraine \\ ${ }^{18}$ Meiji Gakuin University, Faculty of General Education, Yokohama, Japan \\ ${ }^{19}$ Lomonosov Moscow State University, Skobeltsyn Institute of Nuclear Physics, \\ Moscow, Russia \\ ${ }^{20}$ Max-Planck-Institut für Physik, München, Germany \\ ${ }^{21}$ Department of Physics, University of Oxford, Oxford, United Kingdom \\ ${ }^{22}$ INFN Padova, Padova, Italy \\ ${ }^{23}$ Dipartimento di Fisica e Astronomia dell' Università and INFN, Padova, Italy \\ ${ }^{24}$ Polytechnic University, Tokyo, Japan \\ ${ }^{25}$ Raymond and Beverly Sackler Faculty of Exact Sciences, School of Physics, Tel Aviv University, \\ Tel Aviv, Israel \\ ${ }^{26}$ Department of Physics, Tokyo Institute of Technology, Tokyo, Japan \\ ${ }^{27}$ Università di Torino and INFN, Torino, Italy \\ ${ }^{28}$ Università del Piemonte Orientale, Novara, Italy and INFN, Torino, Italy \\ ${ }^{29}$ Physics and Astronomy Department, University College London, \\ London, United Kingdom \\ ${ }^{30}$ Faculty of Physics, University of Warsaw, Warsaw, Poland
}




\author{
${ }^{31}$ National Centre for Nuclear Research, Warsaw, Poland \\ ${ }^{32}$ Department of Particle Physics and Astrophysics, Weizmann Institute, Rehovot, Israel \\ ${ }^{33}$ Department of Physics, York University, Ontario, Canada M3J 1P3
}

(Received 24 February 2016; published 3 May 2016)

\begin{abstract}
A simultaneous fit of parton distribution functions (PDFs) and electroweak parameters to HERA data on deep inelastic scattering is presented. The input data are the neutral current and charged current inclusive cross sections which were previously used in the QCD analysis leading to the HERAPDF2.0 PDFs. In addition, the polarization of the electron beam was taken into account for the ZEUS data recorded between 2004 and 2007. Results on the vector and axial-vector couplings of the $Z$ boson to $u$ - and $d$-type quarks, on the value of the electroweak mixing angle and the mass of the $W$ boson are presented. The values obtained for the electroweak parameters are in agreement with Standard Model predictions.
\end{abstract}

DOI: 10.1103/PhysRevD.93.092002

\section{INTRODUCTION}

Data on deep inelastic scattering (DIS) of leptons from nucleons have been used for many years in many ways to test the Standard Model (SM) of the electroweak and strong interactions [1] and have been fundamental in unravelling the structure of nucleons. The electron-proton, $e p$, collider HERA extended the reach in the four-momentum-transfer squared, $Q^{2}$, and in Bjorken $x$ by several orders of magnitude with respect to previous fixed-target experiments [2]. At HERA, the values of $Q^{2}$ extend up to $50000 \mathrm{GeV}^{2}$, where the $Z$-exchange contribution is comparable to that of the photon exchange. This, together with the longitudinal polarization of the electrons ${ }^{1}$ in the beam, have made a significant test of the couplings of the $Z$ to the

\footnotetext{
${ }^{a}$ Also at INFN, Roma, Italy.

${ }^{\mathrm{b}}$ Also at Sant Longowal Institute of Engineering and Technology, Longowal, Punjab, India.

${ }^{\mathrm{c}}$ Also at Sri Guru Granth Sahib World University, Fatehgarh Sahib, India.

${ }^{\mathrm{d}}$ Also at Agensi Nuklear Malaysia, 43000 Kajang, Bangi, Malaysia.

${ }^{\mathrm{e}}$ Also at Rockefeller University, New York, NY 10065, USA.

${ }^{\mathrm{f}}$ Also at European X-ray Free-Electron Laser facility $\mathrm{GmbH}$, Hamburg, Germany.

${ }^{\mathrm{g}}$ Also at University of Liverpool, United Kingdom.

${ }^{\mathrm{h}}$ Also at Tel Aviv University, Israel.

${ }^{\mathrm{i}}$ Also at Physikalisches Institut, Universität Heidelberg, Germany.

${ }^{\mathrm{j}}$ Also at CERN, Geneva, Switzerland.

${ }^{k}$ Also at DESY and University of Oxford.

${ }^{1}$ Also at DESY.

${ }^{\mathrm{m}}$ Also at University of Tokyo, Japan.

${ }^{\mathrm{n}}$ Also at Kobe University, Japan.

${ }^{\circ}$ Member of National Technical University of Ukraine, Kyiv

Polytechnic Institute, Kyiv, Ukraine.

${ }^{\mathrm{P}}$ Also at DESY CMS group.

${ }^{\mathrm{q}}$ Also at DESY ATLAS group.

${ }^{\mathrm{r}}$ Also at LNF, Frascati, Italy.

${ }^{\mathrm{s}}$ Also at Max Planck Institute for Physics, Munich, Germany.

tAlso at Łódź University, Poland.

${ }^{\text {u} M e m b e r ~ o f ~ Ł o ́ d z ́ ~ U n i v e r s i t y, ~ P o l a n d . ~}$

${ }^{v}$ Also at Polish Air Force Academy in Deblin.
}

quarks possible. The on-shell value of the electroweak mixing angle, $\sin ^{2} \theta_{W}$, and of the mass of the $W$ boson, $M_{W}$, were also determined via a combined QCD and electroweak analysis.

The HERA collider was operated in two phases, HERA I: 1992-2000 and HERA II: 2003-2007. During the HERA II phase, the electron beams were longitudinally polarized to a level between $25 \%$ and $35 \%$. A combination of all ZEUS and $\mathrm{H} 1$ inclusive data for zero polarization was published and subject to a detailed QCD analysis [3], yielding the parton distribution function (PDF) set HERAPDF2.0 and its variants. For the analysis presented here, the ZEUS HERA II data taken at the center-of-mass energy of $318 \mathrm{GeV}$ were used separated into sets with positive and negative polarization as published by the ZEUS collaboration [4-7]. All other data sets were used as originally published by H1 [8-15] and ZEUS [16-23] for unpolarized beams.

\section{STANDARD MODEL FORMALISM}

Inclusive deep inelastic $e p$ scattering can be described in terms of the kinematic variables $Q^{2}, x_{\mathrm{Bj}}$ and $y$. The negative four-momentum-transfer squared, $Q^{2}$, is defined as $Q^{2}=-q^{2}=-\left(k-k^{\prime}\right)^{2}$, where $k$ and $k^{\prime}$ are the fourmomenta of the incoming and the scattered electron, respectively. The Bjorken scaling variable, $x_{\mathrm{Bj}}$, is defined as $x_{\mathrm{Bj}}=Q^{2} / 2 P \cdot q$, where $P$ is the four-momentum of the incoming proton. In the quark-parton model (QPM) the kinematic variable $x_{\mathrm{Bj}}$ is equal to the fractional momentum of the struck quark, $x$. The fraction of the electron energy transferred to the proton in the rest frame of the proton is given by $y=P \cdot q / P \cdot k$. At HERA energies, the masses of the incoming electrons (protons) with energies $E_{e}\left(E_{p}\right)$ can be neglected and the variables $Q^{2}, x_{\mathrm{Bj}}$ and $y$ are related as

\footnotetext{
${ }^{1}$ In this paper, the word "electron" refers to both electrons and positrons, unless otherwise stated.
} 
$Q^{2}=s x_{\mathrm{Bj}} y$, where $s=4 E_{e} E_{p}$ is the square of the electronproton center-of-mass energy.

The components of the Standard Model necessary to describe the data are the electroweak (EW) theory and perturbative quantum chromodynamics (pQCD). At leading order, the EW theory supplies the cross sections for electron scattering from partons with electric charge, i.e. the quarks. The EW theory is subject to pQCD corrections, which already at next-to-leading order make the electron scattering sensitive to the gluons in the proton. The dynamics of the partons, quarks and gluons are described via their PDFs. The PDFs provide the probability of finding a given parton with a momentum fraction $x$ for an interaction at a given factorization scale, $\mu_{\mathrm{f}}$, which is usually chosen to be $Q^{2}$. In pQCD, the PDFs evolve with $Q^{2}$ depending on the order of the strong coupling constant, $\alpha_{s}$, at which the perturbative series is truncated. The analysis presented in this paper was performed at nextto-leading order (NLO) in pQCD.

The $e p$ cross sections measured at HERA were published after they were corrected for leading order (LO) quantum-electrodynamic (QED) radiative effects. These are dominated by initial- and final-state photon emission by the electron.

The neutral current (NC) cross section at all orders of pQCD for $e^{ \pm} p$ scattering can be written as [1]

$$
\begin{aligned}
\frac{d^{2} \sigma_{\mathrm{NC}}\left(e^{ \pm} p\right)}{d x_{\mathrm{Bj}} d Q^{2}}= & \frac{2 \pi \alpha^{2}}{x_{\mathrm{Bj}} Q^{4}}\left[Y_{+} \tilde{F}_{2}\left(x_{\mathrm{Bj}}, Q^{2}\right) \mp Y_{-} x \tilde{F}_{3}\left(x_{\mathrm{Bj}}, Q^{2}\right)\right. \\
& \left.-y^{2} \tilde{F}_{L}\left(x_{\mathrm{Bj}}, Q^{2}\right)\right],
\end{aligned}
$$

where $\alpha$ is the fine-structure constant, $Y_{ \pm}=1 \pm(1-y)^{2}$ and $\tilde{F}_{2}\left(x_{\mathrm{Bj}}, Q^{2}\right), x \tilde{F}_{3}\left(x_{\mathrm{Bj}}, Q^{2}\right)$ and $\tilde{F}_{L}\left(x_{\mathrm{Bj}}, Q^{2}\right)$ are generalized structure functions. The sign in front of the $x \tilde{F}_{3}$ term is taken as positive for electrons and negative for positrons.

The $\tilde{F}_{2}$ term in Eq. (1) is dominant at low $Q^{2}$, where only the photon exchange is important. The longitudinal structure function $\tilde{F}_{L}$ is only significant at very low $Q^{2}$ and irrelevant for this analysis. The $x \tilde{F}_{3}$ term starts to contribute significantly to the cross section at $Q^{2}$ values approaching the mass of the $Z$-boson squared, $M_{Z}^{2}$. The latter originates from $\gamma / Z$ interference and $Z$ exchange and results in a decrease (increase) of the $e^{+} p\left(e^{-} p\right)$ cross sections, respectively.

The data were published as reduced cross sections which were defined for $e^{-} p$ and $e^{+} p$ NC scattering as

$$
\begin{aligned}
\sigma_{r, \mathrm{NC}}^{e^{ \pm} p} & =\frac{x_{\mathrm{Bj}} Q^{4}}{2 \pi \alpha_{0}^{2}} \frac{1}{Y_{+}} \frac{d^{2} \sigma\left(e^{ \pm} p\right)}{d x_{\mathrm{Bj}} d Q^{2}} \\
& =\tilde{F}_{2}\left(x_{\mathrm{Bj}}, Q^{2}\right) \mp \frac{Y_{-}}{Y_{+}} x \tilde{F}_{3}\left(x_{\mathrm{Bj}}, Q^{2}\right)-\frac{y^{2}}{Y_{+}} F_{L}\left(x_{\mathrm{Bj}}, Q^{2}\right) .
\end{aligned}
$$

In this definition, the fine-structure constant is fixed to $\alpha_{0}$, i.e. at scale zero. The QED corrections applied to the data use a running $\alpha$ to correct the data accordingly.

The generalized structure functions depend on the longitudinal polarization of the electron beam, which is defined as

$$
P_{e}=\frac{N_{R}-N_{L}}{N_{R}+N_{L}}
$$

where $N_{R}$ and $N_{L}$ are the numbers of right- and left-handed electrons in the beam.

In all orders, the functions $\tilde{F}_{2}^{ \pm}$and $x \tilde{F}_{3}{ }^{ \pm}$can be split into structure-function terms depending on $\gamma$ exchange $\left(F_{2}^{\gamma}\right), Z$ exchange $\left(F_{2}^{Z}, x F_{3}^{Z}\right)$ and $\gamma / Z$ interference $\left(F_{2}^{\gamma Z}\right.$, $\left.x F_{3}^{\gamma Z}\right)$ as

$$
\begin{aligned}
\tilde{F}_{2}^{ \pm}= & F_{2}^{\gamma}-\left(v_{e} \pm P_{e} a_{e}\right) \chi_{Z} F_{2}^{\gamma Z} \\
& +\left(v_{e}^{2}+a_{e}^{2} \pm 2 P_{e} v_{e} a_{e}\right) \chi_{Z}^{2} F_{2}^{Z}, \\
x \tilde{F}_{3}^{ \pm}= & -\left(a_{e} \pm P_{e} v_{e}\right) \chi_{Z} x F_{3}^{\gamma Z} \\
& +\left(2 v_{e} a_{e} \pm P_{e}\left(v_{e}^{2}+a_{e}^{2}\right)\right) \chi_{Z}^{2} x F_{3}^{Z},
\end{aligned}
$$

where $\chi_{Z}$ is the relative strength of $Z$ exchange with respect to photon exchange. These structure functions depend on the vector and axial-vector couplings of the $Z$ boson to the electron. The SM predictions for these couplings are $v_{e}=$ $-1 / 2+2 \sin ^{2} \theta_{W}$ and $a_{e}=-1 / 2$. The on-shell definition of $\sin ^{2} \theta_{W}=1-M_{W}^{2} / M_{Z}^{2}$ was chosen for the analysis. In the on-shell scheme, this definition is valid to all orders and $M_{W}$ becomes

$$
M_{W}=\frac{A_{0}}{\sin ^{2} \theta_{W} \sqrt{(1-\Delta R)}},
$$

where $A_{0}=\sqrt{\pi \alpha_{0} / \sqrt{2} G_{F}}=37.28039 \mathrm{GeV}$ is a constant [24], $G_{F}$ is the Fermi coupling constant and $\Delta R$ accounts for radiative corrections, the running of $\alpha$ and bosonic loop corrections dominated by the influence of the mass of the top quark [24].

The relative strength of $Z$ exchange with respect to $\gamma$ exchange depends on the on-shell $\sin ^{2} \theta_{W}$ and $\Delta R$ as

$$
\chi_{Z}=\frac{1}{\sin ^{2} 2 \theta_{W}} \frac{Q^{2}}{M_{Z}^{2}+Q^{2}} \frac{1}{1-\Delta R},
$$

where $M_{Z}$ is the pole mass of the $Z$ boson. The value of $\chi_{Z}$ is 0.03 at $Q^{2}=185 \mathrm{GeV}^{2}$, the lowest value of $Q^{2}$ for which ZEUS published inclusive NC cross sections with polarized beams and increases to 1.1 at $Q^{2}=$ $50000 \mathrm{GeV}^{2}$. Since from Eqs. (4) and (5) polarization only enters the structure functions via terms proportional to 
$\chi_{Z}$ or $\chi_{Z}^{2}$, it is evident that beam polarization predominantly affects the cross sections at high $Q^{2}$.

Although this analysis was performed at NLO in QCD, the dominant contributions of the data sets can be identified by considering the structure functions in the framework of the QPM. In this framework, the structure functions can be written in terms of sums and differences of the quark and antiquark PDFs as

$$
\begin{gathered}
{\left[F_{2}^{\gamma}, F_{2}^{\gamma Z}, F_{2}^{Z}\right]=\sum_{q}\left[e_{q}^{2}, 2 e_{q} v_{q}, v_{q}^{2}+a_{q}^{2}\right] x(q+\bar{q}),} \\
{\left[x F_{3}^{\gamma Z}, x F_{3}^{Z}\right]=\sum_{q}\left[e_{q} a_{q}, v_{q} a_{q}\right] 2 x(q-\bar{q}),}
\end{gathered}
$$

where $v_{q}$ and $a_{q}$ are the respective vector and axial-vector couplings of the quark $q$ to the $Z$ boson, and $e_{q}$ is the electric charge of the quark. The PDFs of the quarks and antiquarks are denoted $q$ and $\bar{q}$, respectively.

At any order in pQCD, all quarks kinematically accessible at HERA, i.e. all quarks except the top quark, have to be considered in Eqs. (8) and (9), but the sums are dominated by $u$ - and $d$-quark contributions. It is assumed throughout the analysis that all $u$-type quarks have the same couplings, as do all $d$-type quarks. The SM predictions for the couplings are $v_{u}=1 / 2-4 / 3 \sin ^{2} \theta_{W}, a_{u}=1 / 2$ and $v_{d}=-1 / 2+2 / 3 \sin ^{2} \theta_{W}, a_{d}=-1 / 2$.

For most of the HERA phase space, $\chi_{Z}^{2} \ll \chi_{Z}$ and thus the influence of pure $Z$ exchange is small. In addition, $v_{e} \approx$ 0.04 is small. Thus in Eqs. (4) and (5) the axial-vector couplings are determined predominantly through the term $-a_{e} \chi_{Z} x F_{3}^{\gamma Z}$ and the vector couplings through the term $-P_{e} a_{e} \chi_{Z} F_{2}^{\gamma Z}$. Thus the data obtained with polarized electron beams are crucial for a precise determination of the vector couplings. Nevertheless, it is the combination of all data that provides the final precision.

The charged current (CC) cross sections provide direct information on $M_{W}$. Taking polarization into account, they can be written as

$$
\begin{aligned}
\frac{d^{2} \sigma_{\mathrm{CC}}\left(e^{+} p\right)}{d x_{\mathrm{Bj}} d Q^{2}}= & \left(1+P_{e}\right) \frac{G_{F}^{2} M_{W}^{4}}{2 \pi x_{\mathrm{Bj}}\left(Q^{2}+M_{W}^{2}\right)^{2}} \\
& \times x\left[(\bar{u}+\bar{c})+(1-y)^{2}(d+s+b)\right],
\end{aligned}
$$

$$
\begin{aligned}
\frac{d^{2} \sigma_{\mathrm{CC}}\left(e^{-} p\right)}{d x_{\mathrm{Bj}} d Q^{2}}= & \left(1-P_{e}\right) \frac{G_{F}^{2} M_{W}^{4}}{2 \pi x_{\mathrm{Bj}}\left(Q^{2}+M_{W}^{2}\right)^{2}} \\
& \times x\left[(u+c)+(1-y)^{2}(\bar{d}+\bar{s}+\bar{b})\right] .
\end{aligned}
$$

It follows from Eq. (6) that the coupling $G_{F}$ can be rewritten in terms of $\sin ^{2} \theta_{W}$ and $M_{W}$ as

$$
G_{F}=\frac{\pi \alpha_{0}}{\sqrt{2} \sin ^{2} \theta_{W} M_{W}^{2}} \frac{1}{1-\Delta R} .
$$

Substituting $G_{F}$ into Eqs. (10) and (11) parametrizes the dependence of the CC cross sections on $\sin ^{2} \theta_{W}$.

\section{EXPERIMENTAL SETUP}

The analysis is based on inclusive cross sections for ep scattering published by the $\mathrm{H} 1$ [8-15] and ZEUS [4-7,16-23] collaborations for both the HERA II and HERA I periods. A description of all data sets, including their respective integrated luminosities was published previously [3]. All data sets were taken as input individually; data sets were not combined, in contrast to the HERAPDF2.0 analysis.

Polarized beams were available for the HERA II period from 2003 to 2007 when the electron beam energy was $E_{e}=27.5 \mathrm{GeV}$ and the proton beam was $E_{p}=920 \mathrm{GeV}$, corresponding to a center-of-mass energy of $318 \mathrm{GeV}$. The information on beam polarization was used in this analysis for the corresponding ZEUS HERA II data sets; the H1 HERA II data sets were used as published for zero polarization. The kinematic range of these ZEUS HERA

\begin{tabular}{|c|c|c|c|c|c|c|c|c|c|c|}
\hline \multicolumn{2}{|c|}{ Data set } & \multicolumn{2}{|c|}{$x_{\mathrm{Bj}}$} & \multicolumn{2}{|c|}{$Q^{2}\left[\mathrm{GeV}^{2}\right]$} & \multirow[t]{2}{*}{$e^{+} / e^{-}$} & \multirow[b]{2}{*}{ Points } & \multirow{2}{*}{$\begin{array}{c}\mathcal{L} \\
\mathrm{pb}^{-1}\end{array}$} & \multirow[t]{2}{*}{$P_{e}$} & \multirow[t]{2}{*}{ Reference } \\
\hline Process & Year & From & To & From & To & & & & & \\
\hline $\mathrm{NC}$ & 06-07 & 0.0063 & 0.75 & 185 & 50000 & $e^{+} p$ & 90 & $\begin{array}{l}78.8 \pm 1.4 \\
567+11\end{array}$ & $\begin{array}{l}+0.316 \pm 0.013 \\
-0.353+0.014\end{array}$ & [5] \\
\hline $\mathrm{CC}$ & $06-07$ & 0.0078 & 1.00 & 280 & 50000 & $e^{+} p$ & $\begin{array}{l}35 \\
35\end{array}$ & $\begin{array}{l}75.8 \pm 1.4 \\
56.0 \pm 1.1\end{array}$ & $\begin{array}{l}-0.327 \pm 0.012 \\
-0.358 \pm 0.014\end{array}$ & [7] \\
\hline $\mathrm{NC}$ & $05-06$ & 0.0063 & 0.75 & 185 & 51200 & $e^{-} p$ & $\begin{array}{l}90 \\
90\end{array}$ & $\begin{array}{l}71.2 \pm 1.3 \\
98.7 \pm 1.8\end{array}$ & $\begin{array}{l}+0.289 \pm 0.011 \\
-0.262 \pm 0.011\end{array}$ & [4] \\
\hline $\mathrm{CC}$ & 04-06 & 0.010 & 1.00 & 200 & 60000 & $e^{-} p$ & $\begin{array}{l}34 \\
37\end{array}$ & $\begin{array}{r}71.0 \pm 1.3 \\
104.0 \pm 1.9\end{array}$ & $\begin{array}{l}+0.296 \pm 0.011 \\
-0.267 \pm 0.011\end{array}$ & [6] \\
\hline
\end{tabular}
II data, see Table I, is $185<Q^{2}<51200 \mathrm{GeV}^{2}, 0.0063<$ $x_{\mathrm{Bj}}<0.75$ for $\mathrm{NC}$ and $200<Q^{2}<60000 \mathrm{GeV}^{2}$, $0.0078<x_{\mathrm{Bj}}<1.0$ for CC interactions.

The electron beam in HERA became naturally transversely polarized through the Sokolov-Ternov effect [25].

TABLE I. The four ZEUS data sets, for which polarization was taken into account. 
The characteristic buildup time in HERA was approximately 40 minutes. Spin rotators on either side of the ZEUS detector changed the transverse polarization of the beam into longitudinal polarization in front of the interaction point and subsequently back to transverse polarization. The electron-beam polarization was measured using two independent polarimeters, the transverse polarimeter (TPOL) [26,27] and the longitudinal polarimeter (LPOL) [28]. Both devices exploited the spin-dependent cross section for Compton scattering of circularly polarized photons from electrons. The luminosity and polarization measurements were made over time scales that were much shorter than the polarization buildup time.

The total integrated luminosity for the ZEUS HERA II samples is about $300 \mathrm{pb}^{-1}$. The data were almost evenly divided between positive and negative beam polarization. The ZEUS cross sections for polarized electron beams were published previously [4-7]. For this analysis, the polarization values were corrected using the final information on the polarimeters [29]. The relevant data sets and their polarization values are listed in Table I. The polarization values do not differ by more than $0.3 \%$ from the previously published values for any data set. The uncertainties on the integrated luminosities for all ZEUS HERA II samples were also reevaluated using the final understanding of the luminosity system [30]. The uncertainty is $1.8 \%$ for almost all data taking periods. The uncorrelated part of this uncertainty is $1 \%$.

\section{COMBINED QCD AND EW ANALYSIS}

The analysis presented here was performed at NLO in QCD. The DGLAP [31-35] formalism was used to describe the evolution of the PDFs with $Q^{2}$. The PDFs were parametrized at a starting scale of $1.9 \mathrm{GeV}^{2}$. The analysis followed the method used to extract the set of PDFs called HERAPDF2.0 [3] and its variants. The cross sections as predicted by perturbative QCD were fitted to the measured cross sections and PDF parameters were determined through $\chi^{2}$ minimization. The fits were performed with the ZEUSFITTER package ${ }^{2}$ and cross-checked with the HERAFITTER [37] package.

To extract electroweak parameters, either the couplings of the $Z$ boson to the $u$ - and $d$-type quarks or $\sin ^{2} \theta_{W}$ and $M_{W}$ were additional free parameters in the fit. The resulting PDFs are called ZEUS-EW.

The PDG14 [24] value $M_{Z}=91.1876 \mathrm{GeV}$ was used throughout the analysis. The PDG14 on-shell value of $\sin ^{2} \theta_{W}=0.22333$ [24] and the corresponding SM couplings of the $Z$ boson to $u$ - and $d$-type quarks were used unless these quantities were free parameters in the fits. The

\footnotetext{
${ }^{2}$ The ZEUSFITTER package was previously used to extract the PDF sets of HERAPDF1.0 [36] and to cross-check the HERAPDF2.0 fits.
}

vector couplings of the $Z$ boson to electrons were calculated with the PDG14 on-shell value of $\sin ^{2} \theta_{W}$ and kept fixed throughout the analysis unless $\sin ^{2} \theta_{W}$ was a free parameter. In that case, all couplings of the $Z$ were recalculated according to the SM formulas. The PDG14 value of $M_{W}=80.385 \mathrm{GeV}$ was used unless $M_{W}$ was a free parameter. The PDG14 value of $G_{F}=1.1663787 \times$ $10^{-5} \mathrm{GeV}^{-2}$ was used unless $M_{W}$ or $\sin ^{2} \theta_{W}$ were free parameters in the fit, see Eq. (12).

Only data with $Q^{2} \geq 3.5 \mathrm{GeV}^{2}$ were considered in the analysis. This gives 2942 cross-section points, of which 501 (360 NC and 141 CC) are cross sections measured by ZEUS for polarized beams. Detailed information on these ZEUS data on cross sections for polarized beams are given ${ }^{3}$ in Table I. The number of cross sections used as input to the analysis presented here is much larger than for HERAPDF2.0, because the data sets from ZEUS and H1 were not combined and, in addition, polarization was considered for the ZEUS data sets as listed in Table I doubling the cross-section values for these data sets.

All QCD parameters and settings entering the analysis were chosen as for HERAPDF2.0 unless explicitly stated. The experimental uncertainty, denoted "experimental/fit" in the following, is the uncertainty determined by the fit using the Hessian method. The model uncertainties were computed exactly as for HERAPDF2.0, except for the strange-sea contribution, which was assumed to be a fixed fraction of the $d$-type sea.

The PDFs parametrized are the gluon distribution, $x g$, and the quark distributions in the general form

$$
x f(x)=A x^{B}(1-x)^{C}\left(1+D x+E x^{2}\right) .
$$

The quark distributions are the valence-quark distributions, $x u_{v}, x d_{v}$, and the $u$-type and $d$-type antiquark distributions, $x \bar{U}, x \bar{D}$. The relations $x \bar{U}=x \bar{u}$ and $x \bar{D}=x \bar{d}+x \bar{s}$ are assumed at the starting scale. A detailed discussion on this parametrization ansatz can be found in the HERAPDF2.0 publication [3]. A slight deviation from the HERAPDF2.0 analysis is the reduction from 14 to 13 PDF parameters as described below.

The parametrization of the proton PDFs chosen for ZEUS-EW is

$$
\begin{gathered}
x g(x)=A_{g} x^{B_{g}}(1-x)^{C_{g}}-A_{g}^{\prime} x^{B_{g}^{\prime}}(1-x)^{C_{g}^{\prime}}, \\
x u_{v}(x)=A_{u_{v}} x^{B_{u_{v}}}(1-x)^{C_{u_{v}}}\left(1+E_{u_{v}} x^{2}\right), \\
x d_{v}(x)=A_{d_{v}} x^{B_{d_{v}}}(1-x)^{C_{d_{v}}}, \\
x \bar{U}(x)=A_{\bar{U}} x^{B_{\bar{U}}}(1-x)^{C_{\bar{U}}},
\end{gathered}
$$

\footnotetext{
${ }^{3}$ The data sets were listed as ZEUS NC and ZEUS CC for HERA II $E_{p}=920 \mathrm{GeV}$ in Table I of a previous publication [3].
} 


$$
x \bar{D}(x)=A_{\bar{D}} x^{B_{\bar{D}}}(1-x)^{C_{\bar{D}}} .
$$

The normalization parameters, $A_{u_{v}}, A_{d_{v}}, A_{g}$, are constrained by the quark-number sum rules and the momentum sum rule. The parameters $B_{\bar{U}}$ and $B_{\bar{D}}$ were replaced by a single $B$ parameter for the sea distributions. The strangequark distribution is expressed as an $x$-independent fraction, $f_{s}$, of the $d$-type sea, $x \bar{s}=0.4 x \bar{D}$ at the starting scale. The parameter $C_{g}^{\prime}$ is fixed to $C_{g}^{\prime}=25$ [38]. The reduction to 13 parameters was implemented by replacing $x \bar{U}(x)=$ $A_{\bar{U}} x^{B_{\bar{U}}}(1-x)^{C_{\bar{U}}}\left(1+D_{\bar{U}} x\right)$ used for HERAPDF2.0 with Eq. (17). The reduction to $13 \mathrm{PDF}$ parameters for ZEUS-EW greatly improved the stability of the fits necessary to determine the parametrization uncertainties.

A 13-parameter fit with fixed SM $Z$ couplings, $\sin ^{2} \theta_{W}$ and $M_{W}$, called ZEUS-13p, was performed as a reference. A fit with $13+4$ parameters, called ZEUS-EW-Z, was used to extract the four couplings of the $Z$ to $u$ - and $d$-type quarks. Two $(13+1)$-parameter fits called ZEUS-EW-S and ZEUS-EW-W were used to extract $\sin ^{2} \theta_{W}$ and $M_{W}$ separately, while keeping the other one fixed. In addition, a $(13+2)$-parameter fit called ZEUS-EW-S-W was performed to extract simultaneously $\sin ^{2} \theta_{W}$ and $M_{W}$. As cross-checks, fits in which the PDF parameters were fixed to ZEUS-13p and only the electroweak parameters were allowed to vary were also performed.

The parametrization uncertainties for all fits were obtained by adding extra $D$ and $E$ parameters one by one to the fit. It was checked whether this caused a significant change in the result on the EW parameters. It turned out that only adding back the parameter $D_{\bar{U}}$ or adding the parameter $D_{g}$ resulted in significant differences. If a $(14+4)$-parameter fit including the parameter $D_{\bar{U}}$ would have been chosen for ZEUS-EW, the determination of the parametrization uncertainties would have required $(15+4)$-parameter fits. Such fits were found to be too unstable to provide reliable uncertainties.

The parameter $D_{\bar{U}}$ was added for the extraction of HERAPDF2.0 because it reduced the overall $\chi^{2}$ by about 0.005 per degree of freedom to $1357 / 1131=1.200$. The $\chi^{2}$ per degree of freedom of ZEUS-13p is $3275 / 2929=1.118$. If $D_{\bar{U}}$ would have been added as a 14th parameter, a reduction of $\chi^{2}$ similar to the reduction for HERAPDF2.0 would have been obtained. However, the instability of the $(15+4)$-parameter fits was considered to outweigh this minimal gain in $\chi^{2}$. The $\chi^{2} /$ dof values of all ZEUS-EW fits are similar to the values for ZEUS-13p. As 14-parameter fits were used to evaluate the parametrization uncertainties, the uncertainties associated with the $D_{\bar{U}}$ are included.

All results were cross-checked with fits at NNLO QCD, which yielded compatible results. However, as the EW analysis is partially at LO, see below, a treatment of the PDFs at NLO was considered more consistent, because $\alpha_{s}^{2}$ is of the same order of magnitude as $\alpha$.

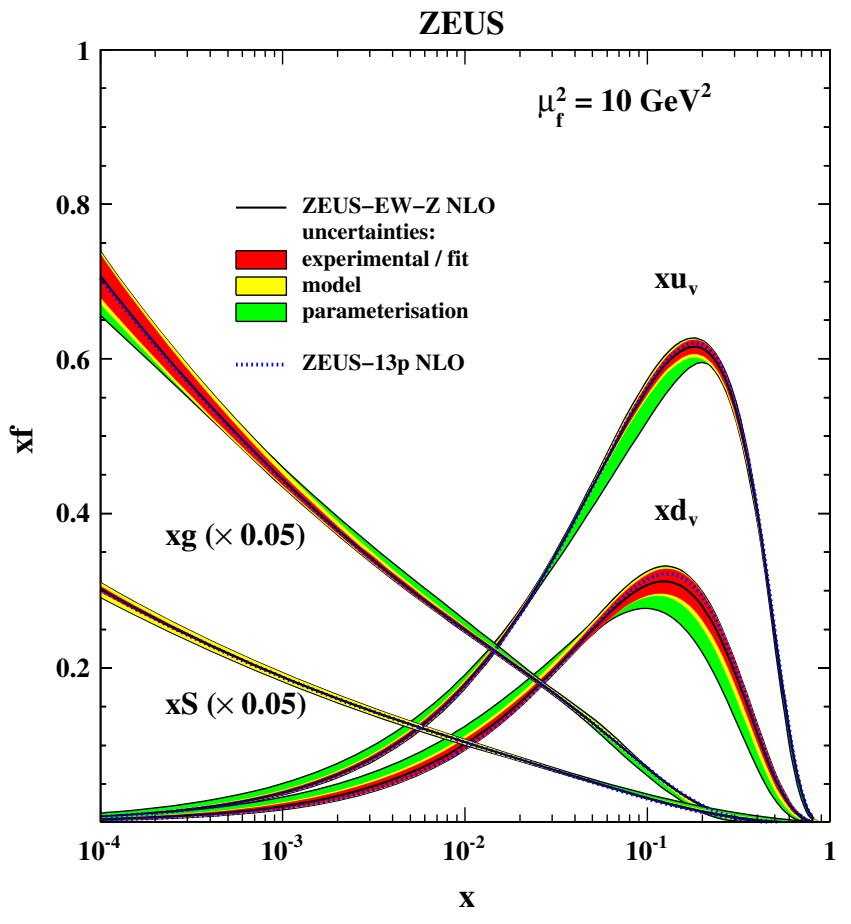

FIG. 1. The PDF set ZEUS-EW-Z with cumulative experimental/fit, model and parametrization uncertainties at the factorization scale $\mu_{\mathrm{f}}^{2}=10 \mathrm{GeV}^{2}$. All positive and negative model uncertainties were added separately in quadrature. The parametrization uncertainty represents an envelope of all individual parametrization uncertainties. Also shown are the central values of the reference fit ZEUS-13p.

The uncertainties on the polarization as listed in Table I were taken into account in all fits presented in this paper. However, it was found that the effect of these uncertainties is negligible compared to the total experimental/fit uncertainty.

As described in Sec. II, the reduced cross sections used as input to the analysis were published by the individual collaborations after QED corrections were applied. These corrections are mostly on the percent level, but reach $15 \%$ for a few cross sections. The correction factors were calculated by producing Monte Carlo data sets for which radiative corrections were either turned on or off for comparison. This was done with the program HeRACLES [39] interfaced to the hadronization programs within the program DJANGOH [40]. However, the two collaborations did not use the HERACLES program with exactly the same options. The ZEUS collaboration only corrected for LO initial- and final-state radiation of the electron. The H1 collaboration included the effects of quark radiation and $Z$ self-energy [8]. ${ }^{4}$ The difference introduced by these extra contributions is, however, always less than $1 \%$ [42]. The H1 collaboration published [8] a cross-check with the

\footnotetext{
${ }^{4}$ The term $Z$ self-energy denotes the influence of vacuum polarization [41].
} 
programs HECTOR [43] and EPRC [41] and concluded that the uncertainties are below $2 \%$ in all of the phase space. In addition, the effect of the exchange of two or more photons between the electron and the quarks, which was not implemented in HeRACLES, was found to be negligible. The H1 collaboration included phase-space-dependent uncertainties in the uncorrelated uncertainties of their published cross sections. The ZEUS collaboration did not assign any uncertainties to their QED corrections. As a cross-check, an extra uncertainty of the size assigned by $\mathrm{H} 1$ was also added to the uncorrelated uncertainties on the ZEUS cross sections for polarized beams. In all cases, the effect on the extracted EW parameters was negligible.

The published cross sections were not corrected for further electroweak effects by either ZEUS or H1. For the analysis presented here, electroweak effects were taken into account through $\Delta R$ as introduced in Eq. (6). It was computed with the program EPRC [41], where weak box diagrams, $\gamma / Z$ interference and $Z$ and $W$ self-energies were taken into account. The running of $\alpha$, relevant for the CC cross sections, is also absorbed in $\Delta R$.

\section{COUPLINGS OF THE $Z$ BOSON TO THE $U$ AND $D$ QUARKS}

To determine the axial-vector and vector couplings of the $Z$ to the $u$ - and $d$-type quarks, $a_{u}, v_{u}, a_{d}, v_{d}$, the QCD predictions depending on the 13 PDF parameters plus the four couplings were fitted simultaneously to the data. The

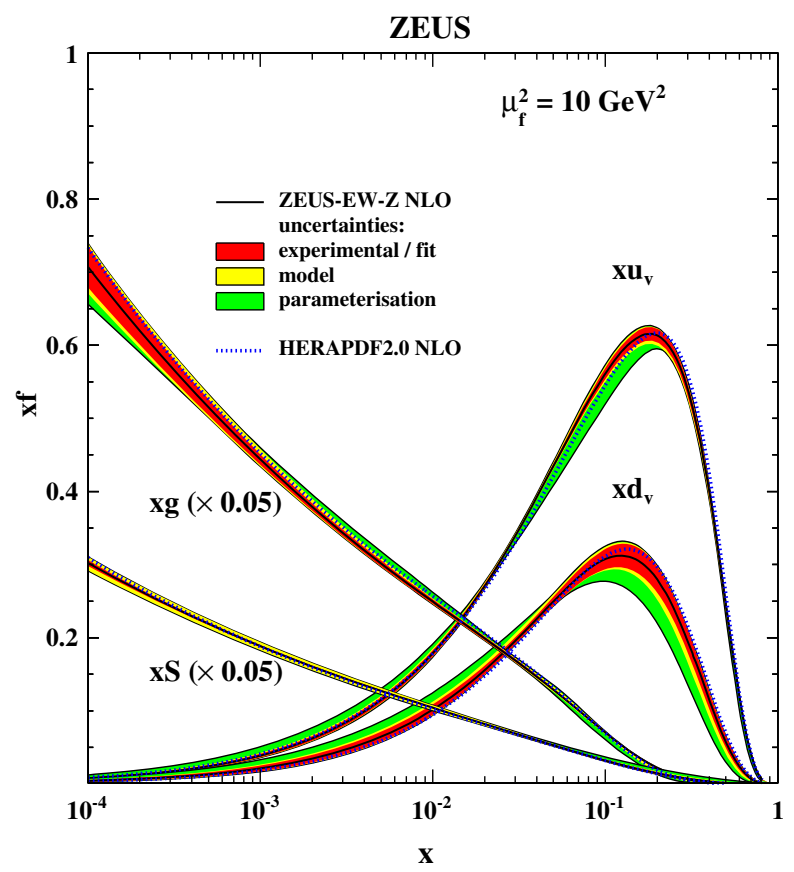

FIG. 2. The PDF set ZEUS-EW-Z with cumulative experimental/fit, model and parametrization uncertainties at the factorization scale $\mu_{\mathrm{f}}^{2}=10 \mathrm{GeV}^{2}$. Also shown are the central values of HERAPDF2.0 NLO. Other details as in Fig. 1. 


\section{ZEUS}

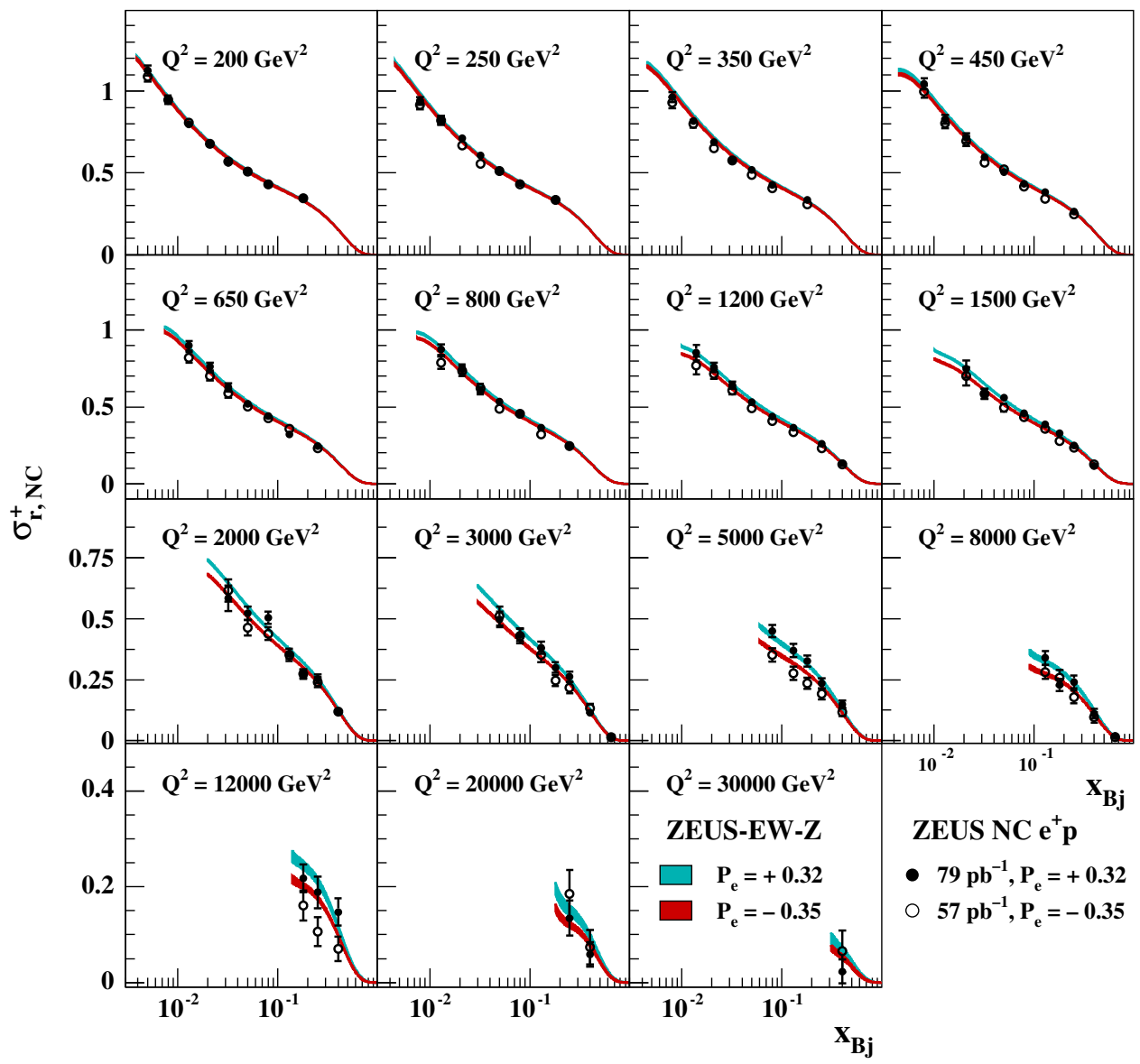

FIG. 3. The predictions of ZEUS-EW-Z compared to the $e^{+} p$ NC DIS reduced cross section $\sigma_{r, N C}^{+}$for positively and negatively polarized beams plotted as a function of $x$ at fixed $Q^{2}$. The closed (open) circles represent the ZEUS data for positive (negative) polarization. The bands indicate the full uncertainty on the predictions of ZEUS-EW-Z.

fit as well as the resulting set of PDFs are called ZEUSEW-Z.

A comparison of the PDFs of ZEUS-EW-Z with full uncertainties to the central values of the PDFs of ZEUS-13p is shown in Fig. 1. Within uncertainties, the PDFs of ZEUS-EW-Z agree well with ZEUS-13p. The freeing of the couplings in the fit has very little influence on the PDF parameters. The full correlation matrix is given as Table II. The small correlation between PDF parameters and couplings is a sign that the PDFs are not absorbing any significant non-SM effects which could show up in the electroweak couplings. A comparison of the PDFs of ZEUS-EW-Z to the PDFs of HERAPDF2.0 is shown in Fig. 2. The PDFs agree well within uncertainties.

The predictions of ZEUS-EW-Z are compared to the ZEUS reduced NC cross sections in Figs. 3 and 4 for $e^{+} p$ and $e^{-} p$ scattering, respectively. In both cases, data with positive and negative beam polarization are shown separately. ZEUS-EW-Z describes the data well.

The values of the couplings were determined in the simultaneous fit as

$$
\begin{aligned}
& a_{u}=+0.500_{-0.05}^{+0.09}(\text { experimental } / \text { fit })_{-0.02}^{+0.04}(\text { model }){ }_{-0.01}^{+0.08}(\text { parametrization }), \\
& \left.a_{d}=-0.56_{-0.14}^{+0.34}(\text { experimental } / \text { fit })_{-0.05}^{+0.11}(\text { model }){ }_{-0.00}^{+0.20} \text { (parametrization }\right) \text {, } \\
& \left.v_{u}=+0.14_{-0.08}^{+0.08}(\text { experimental } / \text { fit })_{-0.02}^{+0.01}(\text { model }){ }_{-0.03}^{+0.00} \text { (parametrization }\right) \text {, } \\
& v_{d}=-0.41_{-0.16}^{+0.24}(\text { experimental } / \text { fit })_{-0.07}^{+0.04}(\text { model }){ }_{-0.08}^{+0.00}(\text { parametrization }) \text {. }
\end{aligned}
$$


ZEUS

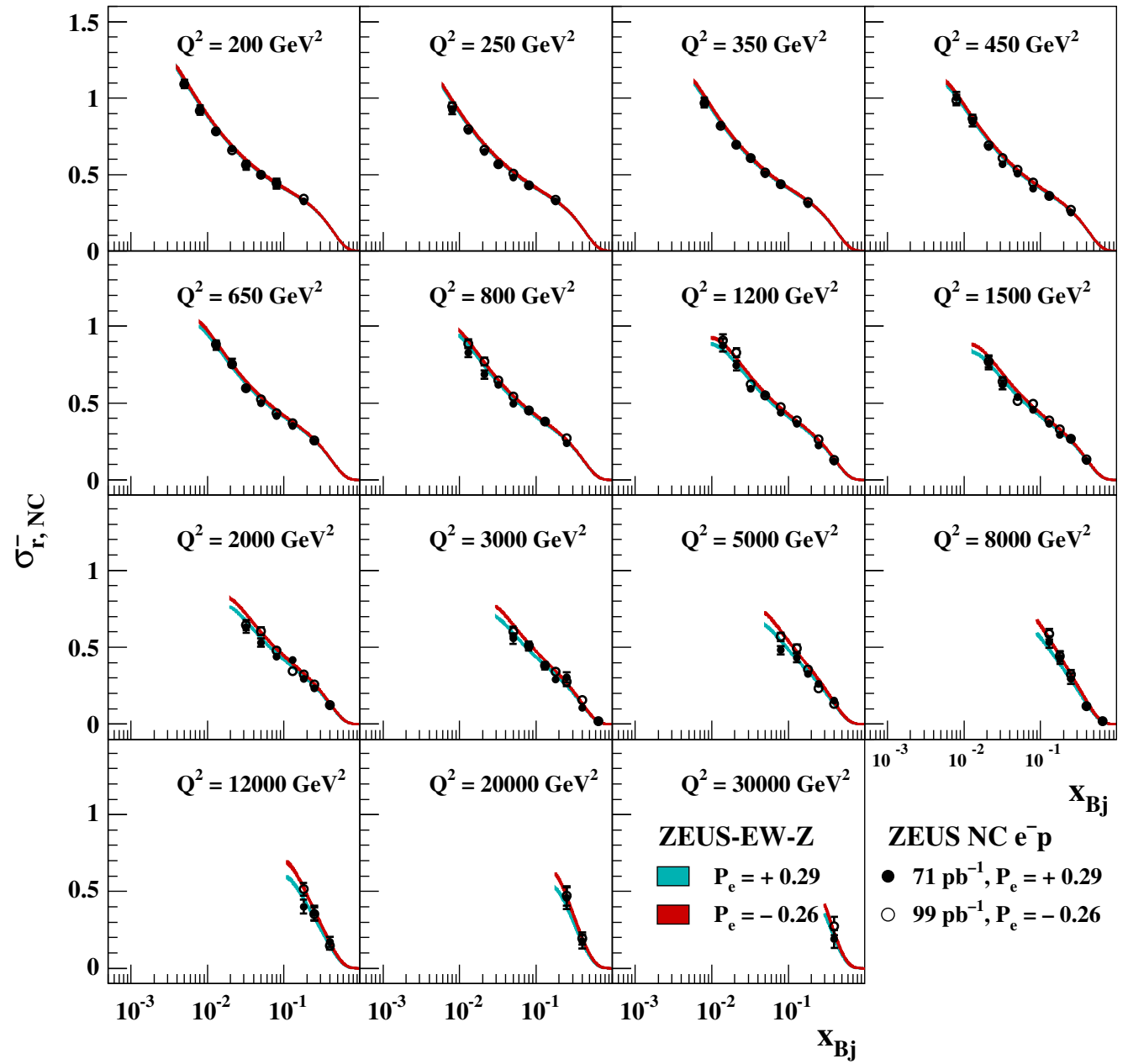

FIG. 4. The predictions of ZEUS-EW-Z compared to the $e^{-} p$ NC DIS reduced cross section $\sigma_{r, N C}^{-}$for positively and negatively polarized beams plotted as a function of $x$ at fixed $Q^{2}$. The closed (open) circles represent the ZEUS data for positive (negative) polarization. The bands indicate the full uncertainty on the predictions of ZEUS-EW-Z.

They are also listed in Table III with their experimental/fit and total uncertainties and compared to SM predictions. Also listed are values obtained in a fit where the only free parameters were the $Z$ couplings and the PDFs were fixed to
ZEUS-13p. These values for the couplings are compatible to those obtained by ZEUS-EW-Z. This cross-check confirms that the determination of the $Z$ couplings is essentially decoupled from the QCD part of the fit.

TABLE III. The results on the axial-vector and vector couplings of the $Z$ boson to $u$ - and $d$-type quarks from ZEUS-EW-Z. Given are the experimental/fit (Exp) and total (Tot) uncertainties. Also listed are results of fits with the PDFs fixed to ZEUS-13p and HERAPDF2.0, HPDF1 and HPDF2, for which only the couplings of the $Z$ were free parameters. The HPDF1 fit was performed with the on-shell value of $\sin ^{2} \theta_{W}$ used in the fit while HPDF2 was performed with the $\sin ^{2} \theta_{W}$ value used for the extraction of HERAPDF2.0. Also listed are the predictions of the SM for the $a$ and $v$ couplings in the on-shell scheme.

\begin{tabular}{|c|c|c|c|c|c|c|c|c|c|c|c|c|}
\hline & $a_{u}$ & Exp & Tot & $a_{d}$ & Exp & Tot & $v_{u}$ & Exp & Tot & $v_{d}$ & Exp & Tot \\
\hline EW-Z & +0.50 & $\begin{array}{l}+0.09 \\
-0.05\end{array}$ & $\begin{array}{l}+0.12 \\
{ }_{-0.05}\end{array}$ & -0.56 & $\begin{array}{l}+0.34 \\
{ }_{-0.14}\end{array}$ & $\begin{array}{l}+0.41 \\
-0.15\end{array}$ & +0.14 & $\begin{array}{l}+0.08 \\
{ }_{-0.08}\end{array}$ & $\begin{array}{l}+0.09 \\
{ }_{-0.09}\end{array}$ & -0.41 & $\begin{array}{l}+0.24 \\
-0.16\end{array}$ & $\begin{array}{l}+0.25 \\
{ }_{-0.20}\end{array}$ \\
\hline $13 p$ & +0.49 & $\begin{array}{l}+0.07 \\
{ }_{-0.04}\end{array}$ & & -0.57 & $\begin{array}{l}+0.30 \\
{ }_{-0.13}\end{array}$ & & +0.15 & $\begin{array}{l}+0.08 \\
-0.08\end{array}$ & & -0.40 & $\begin{array}{l}+0.22 \\
-0.17\end{array}$ & \\
\hline HPDF1 & +0.47 & $\begin{array}{l}+0.06 \\
{ }_{-0.03}\end{array}$ & & -0.62 & $\begin{array}{l}+0.23 \\
{ }_{-0.11}\end{array}$ & & +0.16 & $\begin{array}{l}+0.08 \\
-0.08\end{array}$ & & -0.35 & $\begin{array}{l}+0.22 \\
-0.19\end{array}$ & \\
\hline HPDF2 & +0.49 & $\begin{array}{l}{ }_{-0.03}^{+0.06} \\
{ }_{-0.03}\end{array}$ & & -0.63 & $\begin{array}{l}+0.24 \\
{ }_{-0.11}^{+}\end{array}$ & & +0.15 & $\begin{array}{l}+0.08 \\
-0.08\end{array}$ & & -0.36 & $\begin{array}{l}+0.22 \\
-0.19\end{array}$ & \\
\hline SM & +0.50 & & & -0.50 & & & +0.20 & & & -0.35 & & \\
\hline
\end{tabular}




\section{ZEUS}
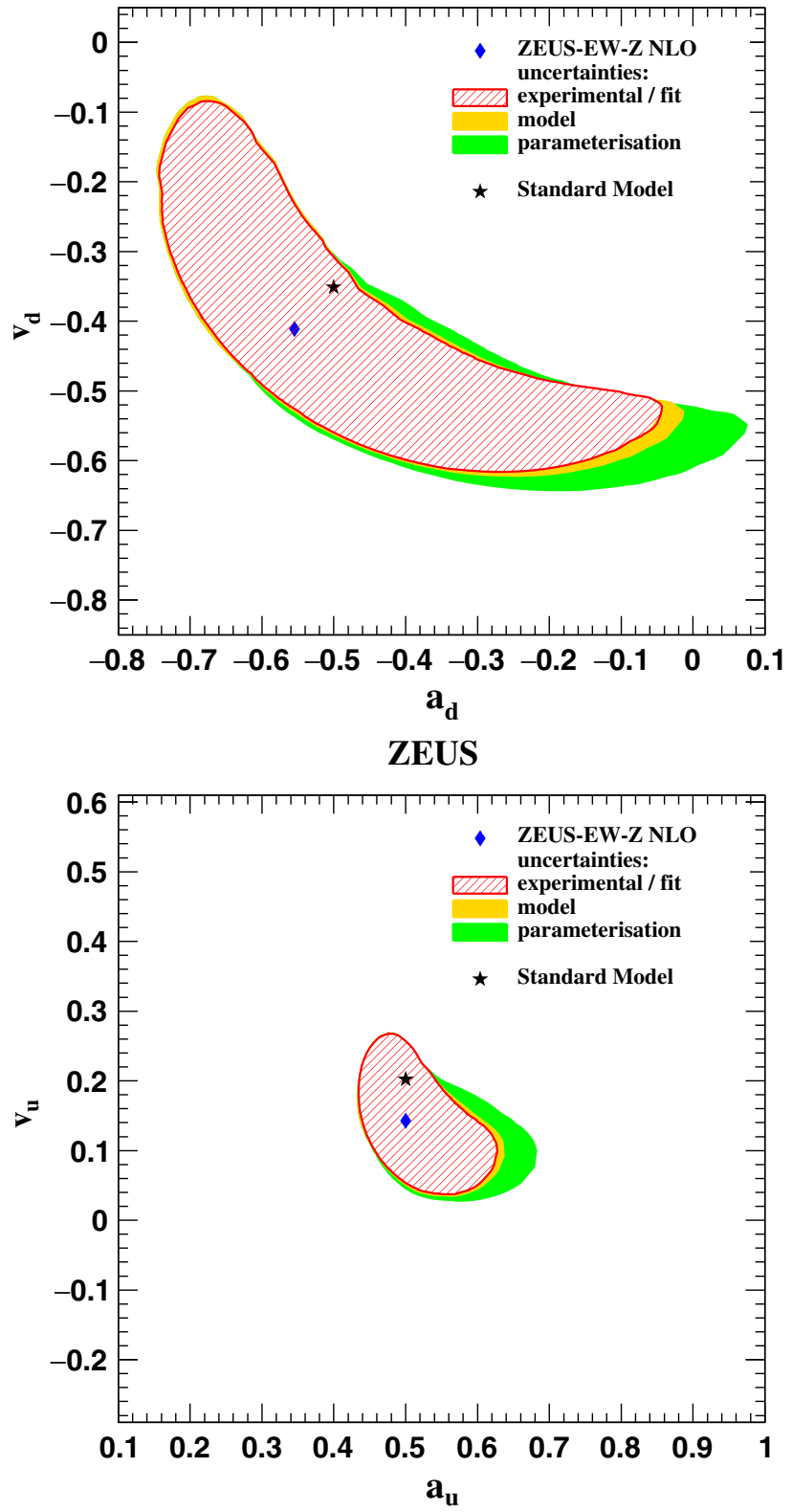

FIG. 5. The $68 \%$ C.L. contours for $\left(a_{d}, v_{d}\right)$ and $\left(a_{u}, v_{u}\right)$ obtained for the ZEUS-EW-Z fit.

Another fit, HPDF1, was performed with the $Z$ couplings free and the PDFs fixed to HERAPDF2.0 NLO. The results, also listed in Table III, are in agreement with ZEUS-EW-Z. It should be noted that HERAPDF2.0 was extracted using a different value of $\sin ^{2} \theta_{W}$. Therefore, a fit HPDF2 using this $\sin ^{2} \theta_{W}$ value was also performed. The result is also listed in Table III. The values agree well within uncertainties with those from the fit using the onshell value.

The correlations between the four couplings obtained in ZEUS-EW-Z are listed as part of Table II. Two-dimensional scans were performed to obtain so-called profile likelihood

\section{ZEUS}

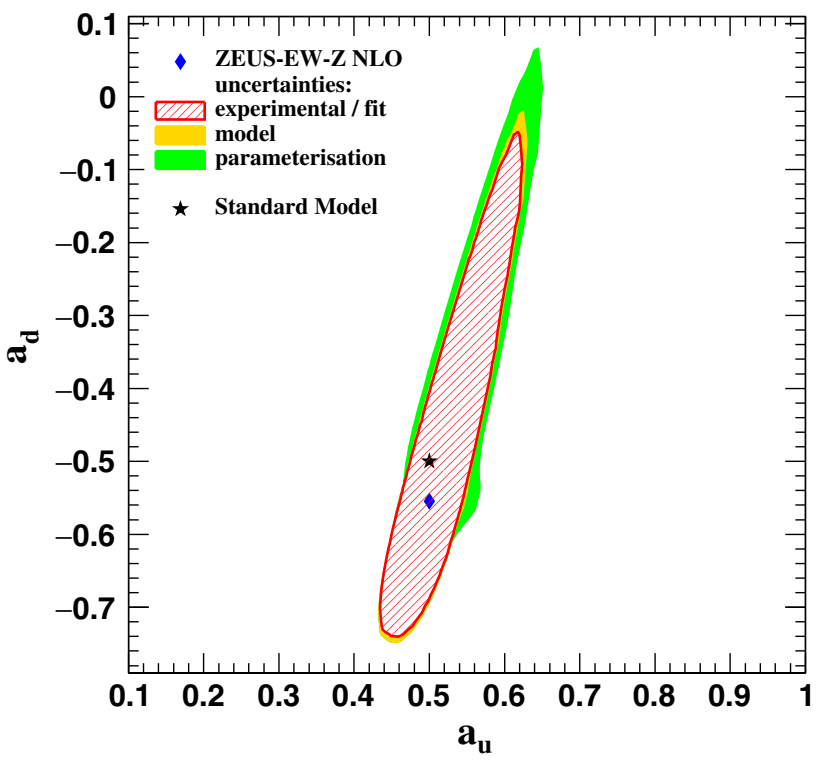

ZEUS

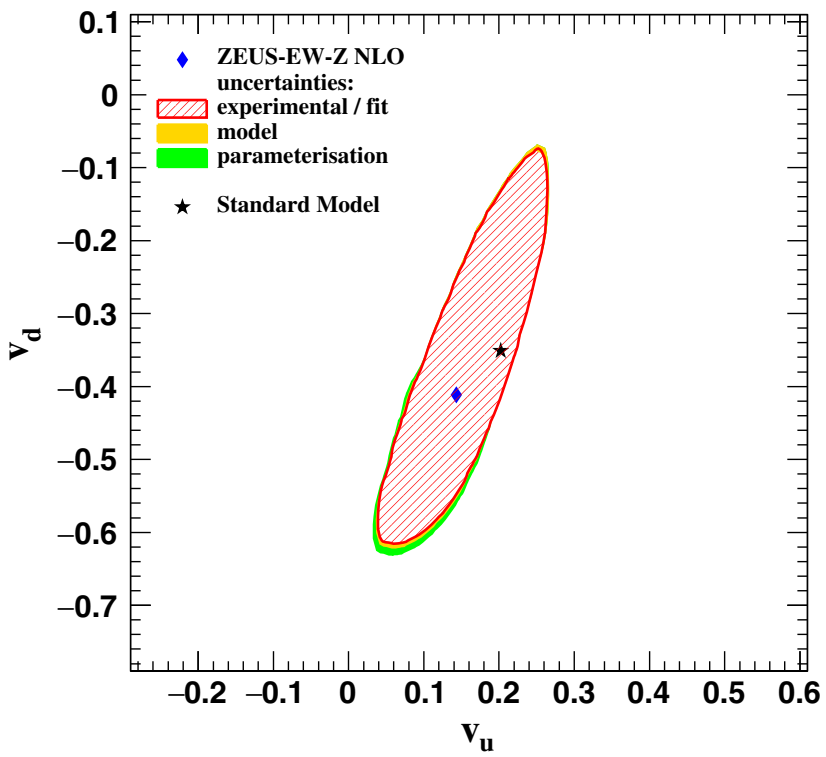

FIG. 6. The $68 \%$ C.L. contours for $\left(a_{u}, a_{d}\right)$ and $\left(v_{u}, v_{d}\right)$ obtained for the ZEUS-EW-Z fit.

contours. The two parameters under investigation were modified in small steps. For each point, a fit was performed to minimize $\chi^{2}$ with respect to all other parameters. The $\chi^{2}$-values thus calculated were used to obtain $68 \%$ C.L. contours. The results for the couplings $a_{u}$, $v_{u}$ and $a_{d}, v_{d}$ are shown in Fig. 5. ${ }^{5}$ Figure 6 shows the $68 \%$ C.L. contour plots for $a_{u}, a_{d}$ and $v_{u}, v_{d}$. This illustration demonstrates very clearly that the HERA

\footnotetext{
${ }^{5}$ Numerical information is available as additional material for this publication.
} 


\section{ZEUS}
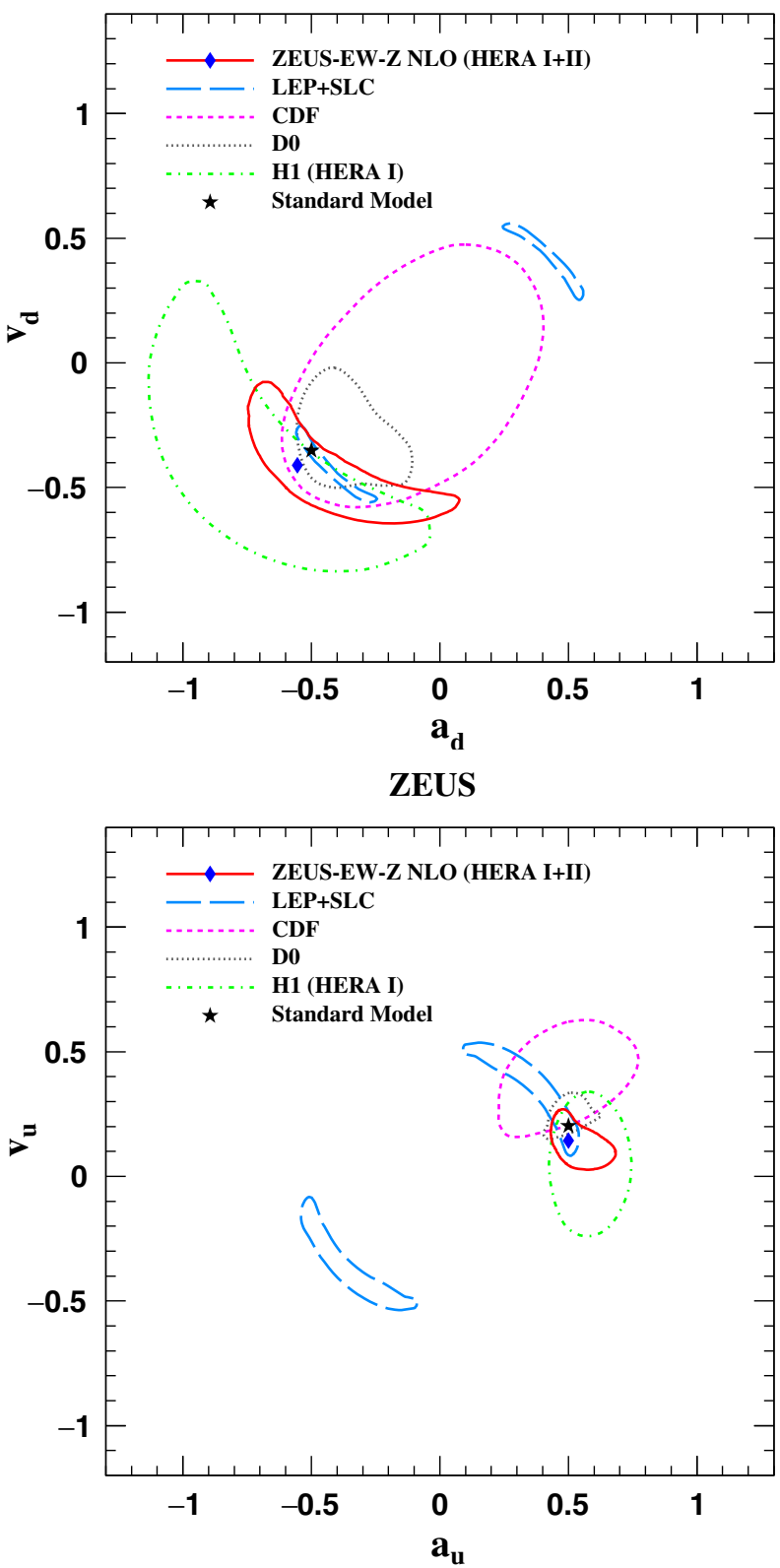

FIG. 7. The $68 \%$ C.L. contours for $\left(a_{d}, v_{d}\right)$ and $\left(a_{u}, v_{u}\right)$ obtained for the ZEUS-EW-Z fit. Also shown are results from LEP (ALEPH, OPAL, L3 and DELPHI) plus SLC (SLD) combined, the Tevatron (CDF and D0), and HERA I (H1).

data constrain the couplings of the $Z$ boson to the $u$ quark significantly better than the couplings to the $d$ quark. All measurements are compatible with the SM. The parametrization uncertainties mostly arise from the $D_{\bar{U}}$ parameter. As this parameter is constrained to be positive, the axial-vector couplings can only increase due to these uncertainties.

The results from ZEUS-EW-Z are compared to other measurements from LEP + SLC [44], the Tevatron [45,46] and from HERA I (H1) [47] in Figs. 7 and 8. The PDG14 value obtained from these measurements is also given in
Fig. 8. The ZEUS results on the axial-vector and vector couplings to $u$-type quarks are the most precise published single values.

\section{ELECTROWEAK MIXING ANGLE AND MASS OF THE $W$ BOSON}

The SM cross sections depend on $\sin ^{2} \theta_{W}$ through three mechanisms:

(1) through $\chi_{z}$, see Eq. (7);

(2) through the normalization factor from Eqs. (10) and (11) with $G_{F}$ rewritten as described in Eq. (12);

(3) through the vector couplings of the $Z$ to the quarks. The $(13+1)$-parameter fit ZEUS-EW-S with $M_{W}$ fixed to the PDG14 value exploits all three dependencies. It yields a value for the on-shell $\sin ^{2} \theta_{W}$ of

$$
\begin{aligned}
\sin ^{2} \theta_{W}= & 0.2252 \pm 0.0011(\text { experimental } / \text { fit })_{-0.0001}^{+0.0003} \\
& \times(\text { model }){ }_{-0.0001}^{+0.0007}(\text { parametrization })
\end{aligned}
$$

The world average in PDG14 for the on-shell value is $\sin ^{2} \theta_{W}=0.22333 \pm 0.00011$ (total). The measurement presented here is slightly high in comparison to the world average. The precision of this result is comparable to the experimental precision achieved in the neutrino sector $[24,48]$. The advantage of the present extraction is that the nuclear effects that have to be taken into account in the analysis of neutrino heavy-target data are not present in $e p$ data. A cross-check was performed with the PDF parameters fixed to ZEUS-EW-13p. The result is $\sin ^{2} \theta_{W}=$ $0.2241 \pm 0.0009$ (experimental/fit), which is compatible with the result from ZEUS-EW-S.

The three mechanisms as listed above influence the result to different degrees. The first mechanism exploits the $\mathrm{NC}$ data. The influence of the second mechanism was tested by fixing $G_{F}$ to its PDG14 value in Eqs. (10) and (11). This removed the influence of the CC data and resulted in an increase of the experimental/fit uncertainty by a factor of three. This demonstrates that both $\mathrm{NC}$ and $\mathrm{CC}$ data contribute significantly to the full precision. The influence of the third mechanism was found to be negligible by fixing the couplings to their SM values.

The PDFs of ZEUS-EW-S are compared to the PDFs of ZEUS-EW-Z in Fig. 9. The two sets of PDFs agree very well. The predictions of ZEUS-EW-S are compared to the reduced CC cross sections in Figs. 10 and 11 for $e^{+} p$ and $e^{-} p$ scattering, respectively. In both cases, data with positive and negative beam polarization are shown separately; ZEUS-EW-S describes the data well.

The $\sin ^{2} \theta_{W}\left(M_{Z}\right)$ value obtained with ZEUS-EW-S can be converted to a value of the effective weak mixing angle [24]. The result is given in Table IV and is shown in Fig. 12 together with the SM prediction [49] for the running of $\sin ^{2} \theta_{W}^{\text {eff }}$. The prediction was computed using the boson and fermion masses and the couplings as listed in PDG14. 


\section{ZEUS}
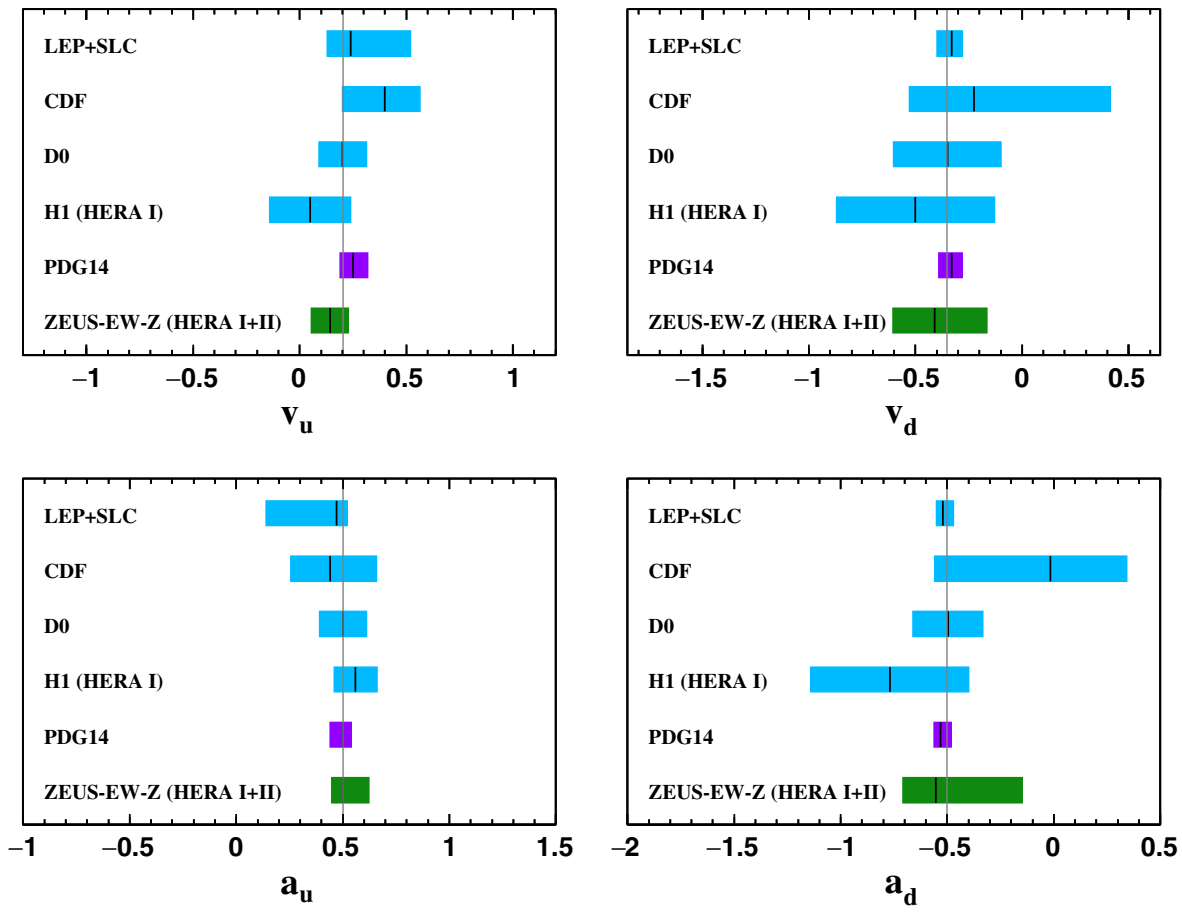

FIG. 8. The values obtained by the ZEUS-EW-Z fit for $a_{d}, a_{u}, v_{d}$ and $v_{u}$ compared to results from LEP (ALEPH, OPAL, L3 and DELPHI) plus SLC (SLD) combined, the Tevatron (CDF and D0), and HERA I (H1) and the world average from these individual measurements as given by PDG14. Vertical black lines in each box indicate central values, the long gray vertical lines indicate the SM predictions. The ZEUS-EW-Z result is given with total uncertainties.

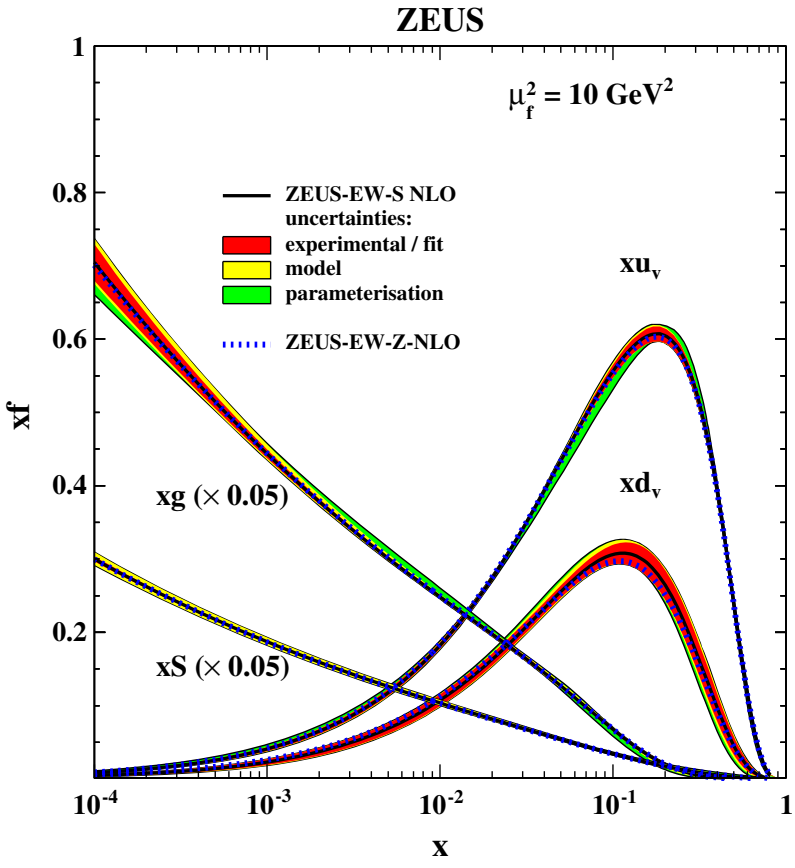

FIG. 9. The PDF set ZEUS-EW-S with cumulative experimental/fit, model and parametrization uncertainties at the factorization scale $\mu_{\mathrm{f}}^{2}=10 \mathrm{GeV}^{2}$. Also shown are the central values of ZEUS-EW-Z. Other details as in Fig. 1.
An additional three fits were performed with the data separated into three $Q^{2}$ bins from 200 to 1000,1000 to 5000 and 5000 to $50000 \mathrm{GeV}^{2}$, using all data available in each range. The scales of the measurement were taken as a log-average $Q^{2}$ value of the given bin. These bins were chosen such that the uncertainties are about equal; cross sections for $Q^{2}<200 \mathrm{GeV}^{2}$ were found to be insensitive to $\sin ^{2} \theta_{W}$. The PDF parameters were fixed to the values determined by the ZEUS-EW-S fit. The resulting on-shell $\sin ^{2} \theta_{W}$ values and the corresponding [34] values of $\sin ^{2} \theta_{W}^{\text {eff }}$ are listed in Table IV together with the values for all data. Also listed are the associated scales. Uncertainties are given for the fits themselves and due to the PDF parameters, model and parametrization uncertainties added in quadrature, as determined by ZEUS-EW$S$. The corresponding effective $\sin ^{2} \theta_{W}$ values are shown together with the result from ZEUS-EW-S in Fig. 12. Also shown are measurements from LEP + SLC [44], D0 [50], CDF [51], CMS [52], ATLAS [53] and LHCb [54], all at the scale of the $Z$ mass, as well as a fixed-target neutrino-nucleon measurement from $\mathrm{NuTeV}$ [48], a fixed target electron-electron measurement from E158 [55] and the result from atomic cesium [56-58] at lower scales. This is the first time that data from a single experimental configuration were used to determine $\sin ^{2} \theta_{W}$ at different 
ZEUS

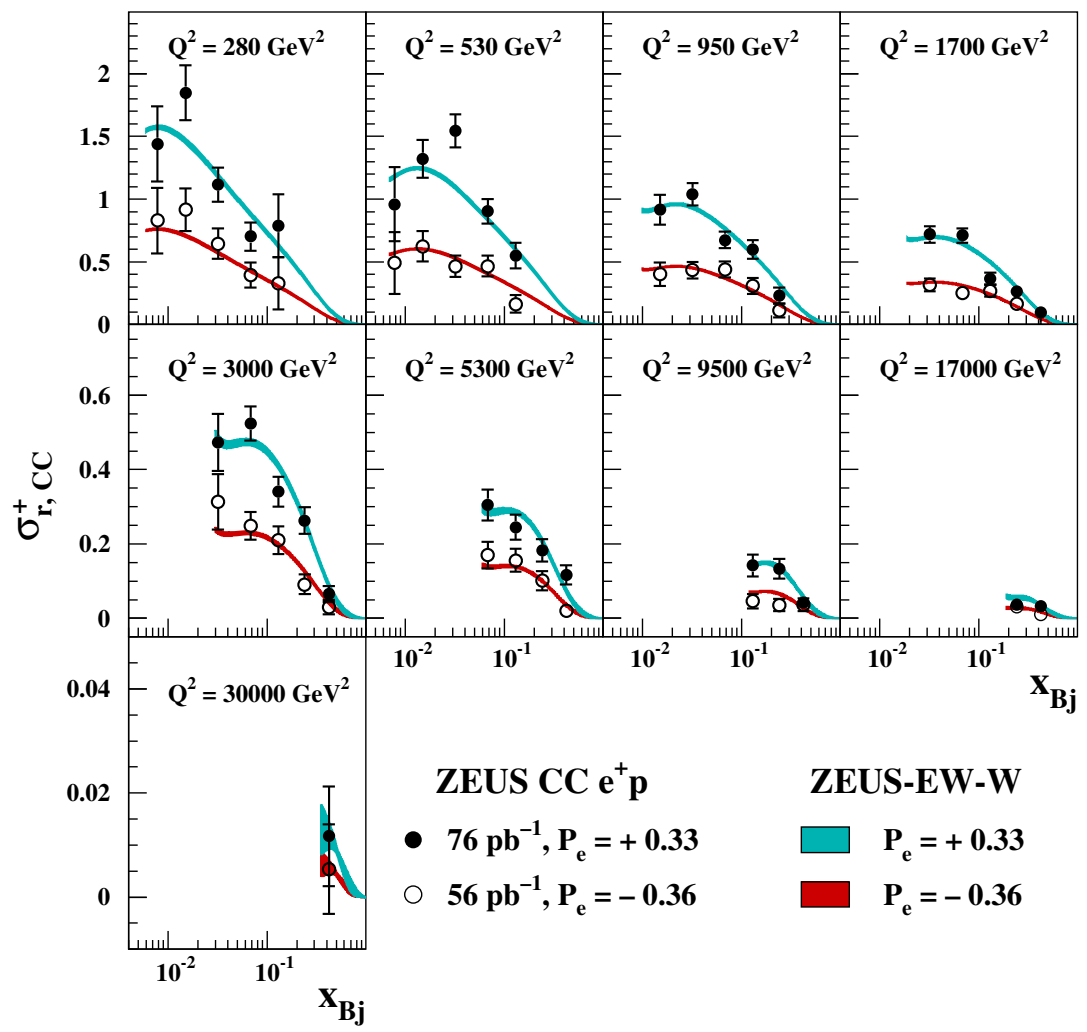

\section{ZEUS}

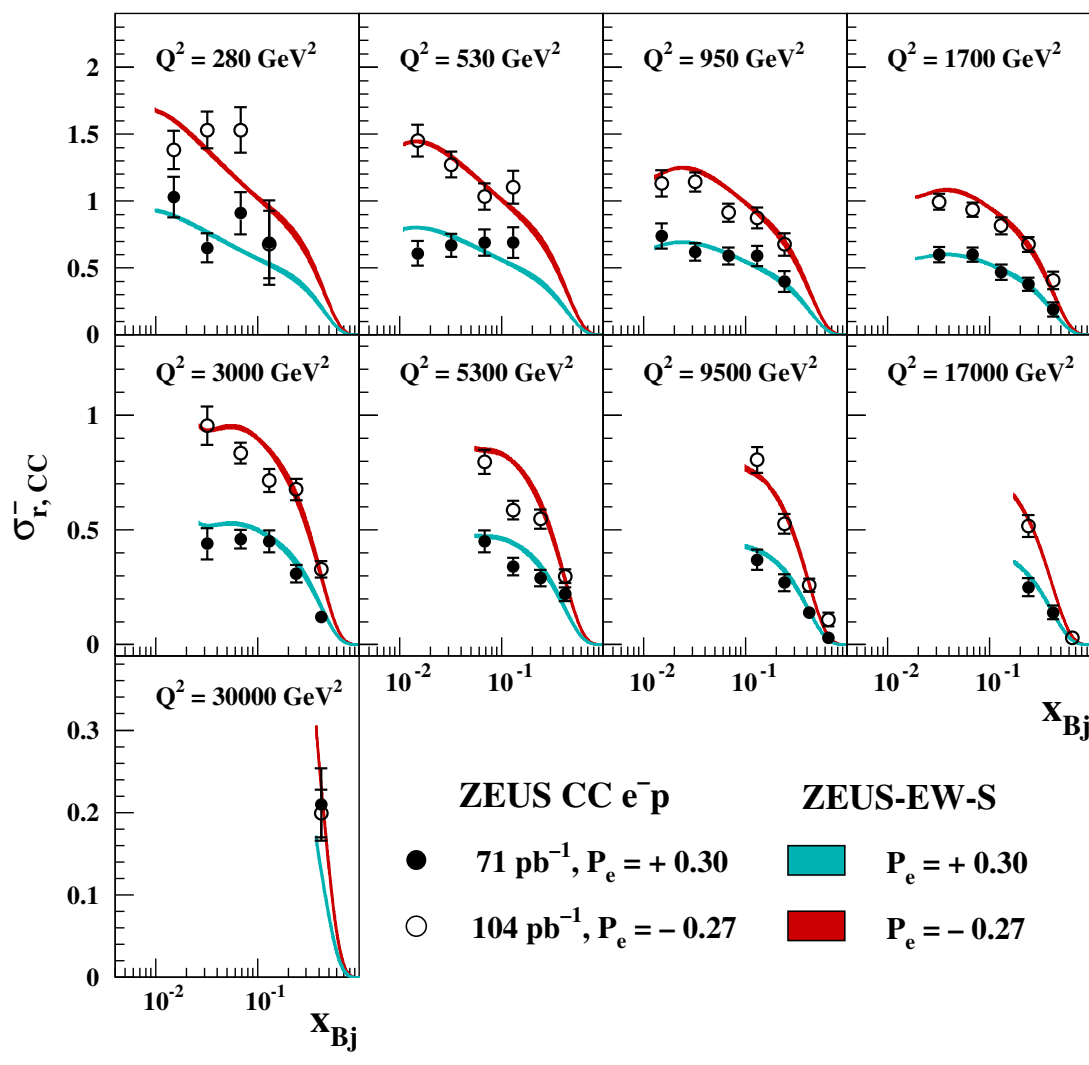

FIG. 10. The predictions of ZEUS-EW-S compared to the $e^{+} p$ CC DIS reduced cross section $\sigma_{r, C C}^{+}$for positively and negatively polarized beams plotted as a function of $x$ at fixed $Q^{2}$. The closed (open) circles represent the ZEUS data for positive (negative) polarization. The bands indicate the full uncertainty on the predictions of ZEUS-EW-S.

FIG. 11. The predictions of ZEUS-EW-S compared to the $e^{-} p$ CC DIS reduced cross section $\sigma_{r, C C}^{-}$for positively and negatively polarized beams plotted as a function of $x$ at fixed $Q^{2}$. The closed (open) circles represent the ZEUS data for positive (negative) polarization. The bands indicate the full uncertainty on the predictions of ZEUS-EW-S. 
TABLE IV. The on-shell and effective values of $\sin ^{2} \theta_{W}$ as determined for three bins in $Q^{2}$ and for all data. Experimental/fit (Exp) uncertainties are given as determined by the one-parameter fits for each bin or ZEUS-EW-S, respectively; model and parametrization uncertainties as determined by ZEUS-EW-S were added in quadrature and are denoted as PDF uncertainties. They are identical for onshell and effective values at the accuracy given.

\begin{tabular}{|c|c|c|c|c|c|c|c|c|}
\hline Bin & $\underset{\left(\mathrm{GeV}^{2}\right)}{Q_{\min }^{2}}$ & $\begin{array}{c}Q_{\max }^{2} \\
\left(\mathrm{GeV}^{2}\right)\end{array}$ & $\begin{array}{l}\text { Scale } \\
(\mathrm{GeV})\end{array}$ & $\begin{array}{c}\sin ^{2} \theta_{W} \\
\text { On shell }\end{array}$ & $\begin{array}{c}\text { Exp } \\
\text { Uncertainties }\end{array}$ & $\begin{array}{l}\sin ^{2} \theta_{W}^{\text {eff }} \\
\text { Effective }\end{array}$ & $\begin{array}{c}\text { Exp } \\
\text { Uncertainties }\end{array}$ & $\begin{array}{c}\text { PDF } \\
\text { Uncertainties }\end{array}$ \\
\hline 1 & 200 & 1000 & 22.3 & 0.2254 & \pm 0.0020 & 0.2352 & \pm 0.0020 & \multirow{4}{*}{$\begin{array}{l}+0.0020 \\
-0.0012 \\
+0.0014 \\
-0.0008 \\
+0.0025 \\
-0.0015 \\
+0.0008 \\
-0.0004\end{array}$} \\
\hline 2 & 1000 & 5000 & 49.9 & 0.2251 & \pm 0.0014 & 0.2339 & \pm 0.0015 & \\
\hline 3 & 5000 & 50000 & 139.8 & 0.2240 & \pm 0.0026 & 0.2323 & \pm 0.0026 & \\
\hline \multicolumn{3}{|c|}{ All data } & $M_{Z}$ & 0.2252 & \pm 0.0011 & 0.2335 & \pm 0.0011 & \\
\hline
\end{tabular}

scales. The result is compatible with the predicted running of the effective $\sin ^{2} \theta_{W}$.

The mass of the $W$ boson was determined by a fit called ZEUS-EW-W with 13 free PDF parameters and, in addition, $M_{W}$ as a free parameter. The $\mathrm{CC}$ cross sections depend directly on $M_{W}$ as shown in Eqs. (10) and (11). However, with $G_{F}$ rewritten as in Eq. (12), the $\mathrm{NC}$ data also contribute to the fit. The value extracted for $M_{W}$ is

$$
\begin{aligned}
M_{W}= & 80.68 \pm 0.28(\text { experimental } / \text { fit })_{-0.01}^{+0.12}(\text { model })_{-0.01}^{+0.23} \\
& \times(\text { parametrization }) \mathrm{GeV} .
\end{aligned}
$$

This $t$-channel determination is in agreement with the PDG14 value of $80.385 \pm 0.015 \mathrm{GeV}$, which is dominated

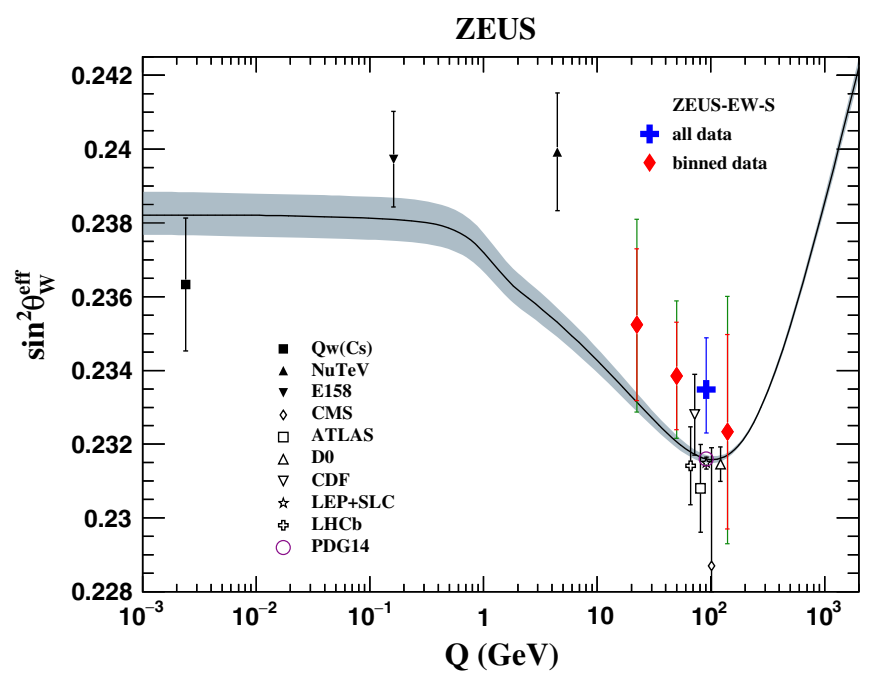

FIG. 12. The scale dependence of $\sin ^{2} \theta_{W}^{\text {eff }}$. The result of ZEUSEW-S is shown as a cross with the error bar representing the total uncertainty. The result in three bins with the 13 PDF parameters fixed to ZEUS-EW-S are shown as diamonds with experimental/ fit and PDF uncertainties (inner and outer error bars). The band represents the SM prediction for the running of the effective $\sin ^{2} \theta_{W}$ for the world average parameters as listed in PDG14. The results from LEP + SLC, CDF, D0, ATLAS, CMS and LHCb are at the scale of the mass of the $Z$ and horizontally displaced for better visibility. The fixed-target experiments NuTeV and E158 and the determination from atomic cesium, $\mathrm{Qw}(\mathrm{Cs})$, provide values at substantially lower scales. by $s$-channel processes. The result presented here is a substantial improvement compared to a result published by H1 using HERA I data [47].

Finally, a fit ZEUS-EW-S-W was performed with 13 free PDF parameters and both $\sin ^{2} \theta_{W}$ and $M_{W}$ as free parameters and with $G_{F}$ rewritten as described in Eq. (12). The resulting values are

$$
\begin{aligned}
\sin ^{2} \theta_{W}= & 0.2293 \pm 0.0031(\text { experimental } / \text { fit })_{-0.0001}^{+0.0005} \\
& \times(\text { model })_{-0.0001}^{+0.0003}(\text { parametrization }) \\
M_{W}= & 79.30 \pm 0.76(\text { experimental } / \text { fit })_{-0.08}^{+0.38} \\
& \times(\text { model }){ }_{-0.10}^{+0.48}(\text { parametrization }) \mathrm{GeV}
\end{aligned}
$$

The uncertainties on $\sin ^{2} \theta_{W}$ and $M_{W}$ are substantially larger than for the determination through ZEUS-EW-S and

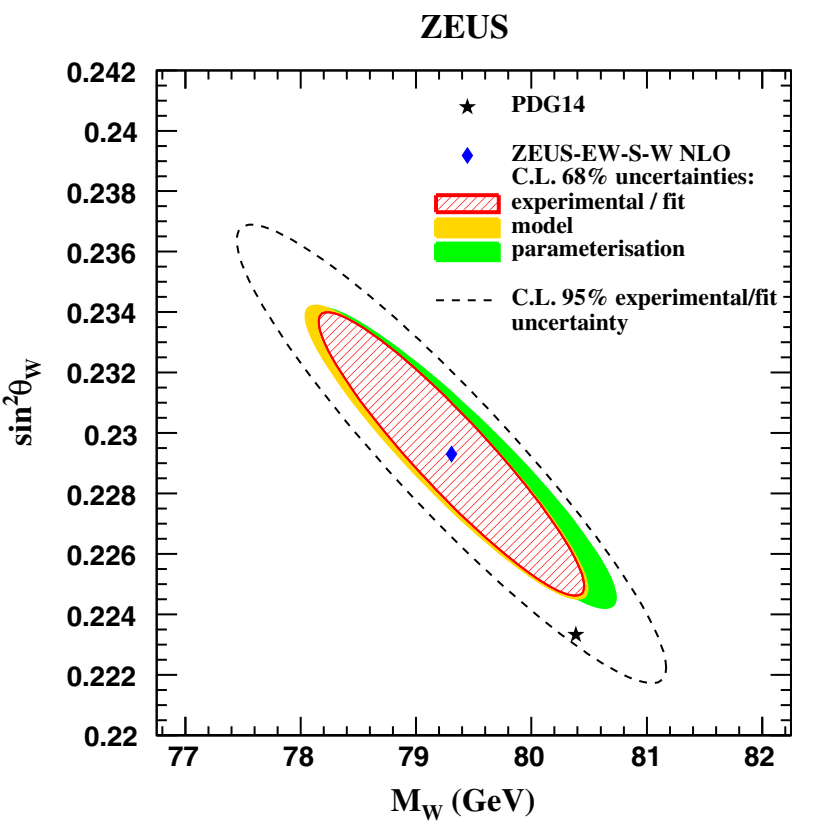

FIG. 13. The cumulative $68 \%$ C.L. contour for $\left(M_{W}, \sin ^{2} \theta_{W}\right)$ for the ZEUS-EW-S-W fit with experimental/fit, model and parametrization uncertainties plotted separately and the 95\% C.L. contour with experimental/fit uncertainties. Also shown is the world average from PDG14. 
ZEUS-EW-W. The values are compatible within these uncertainties. The correlation between the EW parameters and the PDF parameters is small. The correlation between $M_{W}$ and $\sin ^{2} \theta_{W}$ is -0.930 . The $68 \%$ C.L. contour in the $\left(M_{W}, \sin ^{2} \theta_{W}\right)$ plane with experimental/fit, model and parametrization uncertainties plotted separately is shown in Fig. 13. Also shown is the 95\% C.L. contour with experimental/fit uncertainties only. The world average from PDG14 is shown as a reference. The values for $\left(\sin ^{2} \theta_{W}, M_{W}\right)$ are within 2 sigma of the world average.

\section{SUMMARY AND CONCLUSIONS}

A combined QCD and electroweak analysis was performed based on all HERA $e p$ inclusive scattering data, exploiting the beam polarization for ZEUS data taken during the years 2004 to 2007 during the HERA II period with a center-of-mass energy of $318 \mathrm{GeV}$. The kinematic range of these ZEUS HERA II data is $185<Q^{2}<51200 \mathrm{GeV}^{2}$, $0.0063<x_{\mathrm{Bj}}<0.75$ for $\mathrm{NC}$ and $200<Q^{2}<60000 \mathrm{GeV}^{2}$, $0.0078<x_{\mathrm{Bj}}<1.0$ for $\mathrm{CC}$ interactions.

The couplings of the $Z$ boson to $u$ - and $d$-type quarks were determined by a QCD plus EW fit with 13 parameters for the PDFs and four parameters for the $Z$ couplings. The resulting set of PDFs is compatible with a 13-parameter QCD-only fit and HERAPDF2.0. The correlations between the PDF and coupling parameters are small.

The results for the axial-vector and vector coupling of the $Z$ boson to $u$ - and $d$-type quarks are

$$
\begin{aligned}
& a_{u}=+0.50_{-0.05}^{+0.09}(\text { experimental } / \text { fit })_{-0.02}^{+0.04}(\text { model }){ }_{-0.01}^{+0.08}(\text { parametrization }), \\
& \left.a_{d}=-0.56_{-0.14}^{+0.34}(\text { experimental } / \text { fit })_{-0.05}^{+0.11}(\text { model }){ }_{-0.00}^{+0.20} \text { (parametrization }\right) \text {, } \\
& \left.v_{u}=+0.14_{-0.08}^{+0.08} \text { (experimental/fit) }{ }_{-0.02}^{+0.01} \text { (model }\right)_{-0.03}^{+0.00} \text { (parametrization), } \\
& v_{d}=-0.41_{-0.16}^{+0.24}\left(\text { experimental/fit }{ }_{-0.07}^{+0.04}(\text { model }){ }_{-0.08}^{+0.00} \text { (parametrization }\right) \text {. }
\end{aligned}
$$

The values of $M_{W}$ and $\sin ^{2} \theta_{W}$ in the on-shell scheme were extracted with (13+1)-parameter fits. The value extracted for $M_{W}$ is

$$
M_{W}=80.68 \pm 0.28(\text { experimental } / \text { fit })_{-0.01}^{+0.12}(\text { model }){ }_{-0.01}^{+0.23} \text { (parametrization) } \mathrm{GeV} .
$$

The on-shell value of $\sin ^{2} \theta_{W}$ was determined as

$$
\sin ^{2} \theta_{W}=0.2252 \pm 0.0011 \text { (experimental/fit) }{ }_{-0.0001}^{+0.0003} \text { (model) }{ }_{-0.0001}^{+0.0007} \text { (parametrization) }
$$

The determination of $\sin ^{2} \theta_{W}$ is competitive with results obtained in the neutrino sector. In addition, the data were subdivided such that values of the effective $\sin ^{2} \theta_{W}^{\text {eff }}$ for three different values of the scale could be determined. The values of $\sin ^{2} \theta_{W}$ and $M_{W}$ as well as of the couplings of the $Z$ boson are in agreement with Standard Model expectations. The values of the axial-vector and vector couplings of the $Z$ boson to $u$-type quarks presented in this paper are the most precise determination published by a single collaboration.

\section{ACKNOWLEDGMENTS}

We appreciate the contributions to the construction, maintenance and operation of the ZEUS detector of many people who are not listed as authors. The HERA machine group and the DESY computing staff are especially acknowledged for their success in providing excellent operation of the collider and the data-analysis environment. We thank the DESY directorate for their strong support and encouragement. This work has been supported in part by: the Italian National Institute for Nuclear Physics (INFN); the German Federal Ministry for Education and Research (BMBF), under Contract No. 05 H09PDF; HIR Grant No. UM.C/625/1/HIR/149 and UMRG Grants No. RU0062013, RP012A-13AFR and RP012B-13AFR from Universiti Malaya, and ERGS Grant No. ER004-2012A from the Ministry of Education, Malaysia; the National Science Centre, Poland, under Contract No. DEC-2012/06/ M/ST2/00428; the Science and Technology Facilities Council, UK; the German Federal Ministry for Education and Research (BMBF), under Contract No. 05h09GUF, and the SFB 676 of the Deutsche Forschungsgemeinschaft (DFG); the Japanese Ministry of Education, Culture, Sports, Science and Technology (MEXT) and its grants for Scientific Research; RF Presidential Grant No. 3042.2014.2 for the Leading Scientific Schools, Russia; the Israel Science Foundation; the Natural Sciences and Engineering Research Council of Canada (NSERC); the Polish National Science Centre Projects No. DEC-2011/01/B/ ST2/03643 and DEC-2011/03/B/ST2/00220; and the Alexander von Humboldt Foundation. 
[1] A. M. Cooper-Sarkar and R. Devenish, Deep Inelastic Scattering (Oxford University Press, New York, 2011).

[2] H. Abramowicz and A. Caldwell, Rev. Mod. Phys. 71, 1275 (1999).

[3] H. Abramovicz et al. (ZEUS and H1 Collaborations), Eur. Phys. J. C 75, 580 (2015).

[4] S. Chekanov et al. (ZEUS Collaboration), Eur. Phys. J. C 62, 625 (2009).

[5] H. Abramowicz et al. (ZEUS Collaboration), Phys. Rev. D 87, 052014 (2013).

[6] S. Chekanov et al. (ZEUS Collaboration), Eur. Phys. J. C 61, 223 (2009).

[7] H. Abramowicz et al. (ZEUS Collaboration), Eur. Phys. J. C 70, 945 (2010).

[8] F. D. Aaron et al. (H1 Collaboration), J. High Energy Phys. (2012) 061.

[9] V. Andreev et al. (H1 Collaboration), Eur. Phys. J. C 74, 2814 (2014).

[10] F. D. Aaron et al. (H1 Collaboration), Eur. Phys. J. C 71, 1579 (2011).

[11] C. Adloff et al. (H1 Collaboration), Eur. Phys. J. C 13, 609 (2000).

[12] C. Adloff et al. (H1 Collaboration), Eur. Phys. J. C 19, 269 (2001).

[13] C. Adloff et al. (H1 Collaboration), Eur. Phys. J. C 30, 1 (2003).

[14] F. D. Aaron et al. (H1 Collaboration), Eur. Phys. J. C 64, 561 (2009).

[15] F. D. Aaron et al. (H1 Collaboration), Eur. Phys. J. C 63, 625 (2009).

[16] H. Abramowicz et al. (ZEUS Collaboration), Phys. Rev. D 90, 072002 (2014).

[17] J. Breitweg et al. (ZEUS Collaboration), Eur. Phys. J. C 7, 609 (1999).

[18] J. Breitweg et al. (ZEUS Collaboration), Eur. Phys. J. C 12, 411 (2000); 27, 305(E) (2003).

[19] S. Chekanov et al. (ZEUS Collaboration), Eur. Phys. J. C 21, 443 (2001).

[20] S. Chekanov et al. (ZEUS Collaboration), Phys. Lett. B 539, 197 (2002); 552, 308(E) (2003).

[21] S. Chekanov et al. (ZEUS Collaboration), Eur. Phys. J. C 28, 175 (2003).

[22] S. Chekanov et al. (ZEUS Collaboration), Eur. Phys. J. C 32, 1 (2003).

[23] S. Chekanov et al. (ZEUS Collaboration), Phys. Rev. D 70, 052001 (2004).

[24] K. A. Olive et al. (Particle Data Group), Chin. Phys. C 38, 090001 (2014).

[25] A. A. Sokolov and I. M. Ternov, Sov. Phys. Dokl. 8, 1203 (1964).

[26] V. N. Baier and V. A. Khoze, Sov. J. Nucl. Phys. 9, 238 (1969).

[27] D. P. Barber et al., Nucl. Instrum. Methods Phys. Res., Sect. A 329, 79 (1993).

[28] M. Beckmann et al., Nucl. Instrum. Methods Phys. Res., Sect. A 479, 334 (2002).

[29] T. Behnke et al., arXiv:1201.2894.

[30] L. Adamczyk et al., Nucl. Instrum. Methods Phys. Res., Sect. A 744, 80 (2014).
[31] V. N. Gribov and L. N. Lipatov, Sov. J. Nucl. Phys. 15, 438 (1972).

[32] V. N. Gribov and L. N. Lipatov, Sov. J. Nucl. Phys. 15, 675 (1972).

[33] L. N. Lipatov, Sov. J. Nucl. Phys. 20, 94 (1975).

[34] Y. L. Dokshitzer, Sov. Phys. JETP 46, 641 (1977).

[35] G. Altarelli and G. Parisi, Nucl. Phys. B126, 298 (1977).

[36] F. D. Aaron et al. (ZEUS and H1 Collaborations), J. High Energy Phys. (2010) 109, and references therein.

[37] S. Alekhin et al., Eur. Phys. J. C 75, 304 (2015).

[38] A. D. Martin, W. J. Stirling, R. S. Thorne, and G. Watt, Eur. Phys. J. C 63, 189 (2009).

[39] A. Kwiatkowski, H. Spiesberger, and H.-J. Möhring, Comput. Phys. Commun. 69, 155 (1992); also in Proceedings of the Workshop on Physics at HERA, edited by W. Buchmüller and G. Ingelman (DESY, Hamburg, 1991).

[40] G. A. Schuler and H. Spiesberger, in Proceedings of the Workshop on Physics at HERA, edited by W. Buchmüller and G. Ingelman (DESY, Hamburg, Germany, 1991), Vol. 3, p. 1419.

[41] H. Spiesberger, in Proceedings of the Workshop on Future Physics at HERA, edited by G. Ingelman, A. De Roeck, and R. Klanner (DESY, Hamburg, Germany, 1995).

[42] D. Yu. Bardin, Č. Burdík, P. Ch. Christova, and T. Riemann, Z. Phys. C 42, 679 (1989).

[43] A. Arbuzov, D. Bardin, J. Blümlein, L. Kalinovskaya, and T. Riemann, Comput. Phys. Commun. 94, 128 (1996).

[44] G. Abbiendi et al. [ALEPH, DELPHI, L3 and OPAL Collaborations, and SLD Collaboration (LEP Electroweak Working Group, SLD Electroweak and Heavy Flavor Groups)], Phys. Rept. 427, 257 (2006).

[45] V. Abazov et al. (D0 Collaboration), Phys. Rev. D 84, 012007 (2011).

[46] D. Acosta et al. (CDF Collaboration), Phys. Rev. D 71, 052002 (2005).

[47] A. Aktas et al. (H1 Collaboration), Phys. Lett. B 632, 35 (2006).

[48] G. P. Zeller et al. (NuTeV Collaboration), Phys. Rev. Lett. 88, 091802 (2002); 90, 239902(E) (2003).

[49] A. Czarnecki and W. J. Marciano, Int. J. Mod. Phys. A 15, 2365 (2000).

[50] V. Abazov et al. (D0 Collaboration), Phys. Rev. Lett. 115, 041801 (2015).

[51] T. Aaltonen et al. (CDF Collaboration), Phys. Rev. D 88, 072002 (2013); 88, 079905(E) (2013).

[52] S. Chatrchyan et al. (CMS Collaboration), Phys. Rev. D 84, 112002 (2011).

[53] G. Aad et al. (ATLAS Collaboration), J. High Energy Phys. 09 (2015) 049.

[54] R. Aaij et al. (LHCb Collaboration), J. High Energy Phys. 11 (2015) 190.

[55] P. L. Anthony et al. (E158 Collaboration), Phys. Rev. Lett. 95, 081601 (2005).

[56] S. C. Bennett and C. E. Wieman, Phys. Rev. Lett. 82, 2484 (1999).

[57] C. S. Wood et al., Science 275, 1759 (1997).

[58] J. Guena, M. Lintz, and M. A. Bouchiat, Phys. Rev. A 71, 042108 (2005). 